\title{
Is There Any Evidence of Premature, Accentuated and Accelerated Aging Effects on Neurocognition in People Living with HIV? A Systematic Review
}

\author{
Htein Linn Aung ${ }^{1,2,3}$ (D) Maral Aghvinian ${ }^{4} \cdot$ Hetta Gouse $^{5} \cdot$ Reuben N. Robbins $^{6} \cdot$ Bruce J. Brew $^{1,3,7} \cdot$ Limin Mao $^{8} \cdot$ \\ Lucette A. Cysique ${ }^{1,2,3}$
}

Accepted: 24 September 2020 / Published online: 6 October 2020

(c) The Author(s) 2020

\begin{abstract}
Despite evidence of premature, accentuated and accelerated aging for some age-related conditions such as cardiovascular diseases in people living with HIV (PLHIV), the evidence for these abnormal patterns of aging on neurocognition remains unclear. Further, no systematic review has been dedicated to this issue. Using PRISMA guidelines, we searched standard databases (PubMed, EMBASE, CINAHL and PsycINFO). Articles were included if they analyzed and reported the effect of age on neurocognition among PLHIV as one of their major findings, if they were conducted in the combination anti-retroviral therapy era (after 1996) and published in a peer-reviewed journal in English. Quality appraisal was conducted using the Joanna Briggs Institute (JBI) appraisal tools. To systematically target the abnormal patterns of neurocognitive aging, we define premature cognitive aging as significant interaction effect of HIV status and age on cross-sectional neurocognitive test performance covering both the normal and abnormal performance range; accentuated cognitive aging as significant interaction effect of HIV status and age on cross-sectional neurocognitive impairment (NCI) rate, thus covering the abnormal performance range only; accelerated cognitive aging as significant interaction effect of HIV status and age on longitudinal neurocognitive test performance or incidence of NCI. Because these definitions require an age-comparable HIV-negative (HIV-) control group, when no controls were included, we determined the range of the age effect on neurocognitive test performance or NCI among PLHIV. A total of 37 studies originating from the US (26), UK (2), Italy (2), Poland (2), China (2), Japan (1), Australia (1), and Brazil (1) were included. Six studies were longitudinal and 14 included HIV- controls. The quality appraisal showed that 12/37 studies neither used an age-matched HIV-controls nor used demographically corrected cognitive scores. A meta-analysis was not possible because study methods and choice of neurocognitive measurement methods and outcomes were heterogeneous imposing a narrative synthesis. In studies with an HIV- control sample, premature neurocognitive aging was found in $45 \%$ of the cross-sectional analyses (9/20), while accelerated neurocognitive aging was found in $75 \%$ of the longitudinal analyses (3/4). There was no evidence for accentuated aging, but this was tested only in two studies. In studies without an HIV-control sample, the age effect was always present but wide (NCI OR $=1.18-4.8)$. While large sample size (>500) was associated with abnormal patterns of cognitive aging, most of the studies were under powered. Other study characteristics such as longitudinal study design and higher proportion of older participants were also associated with the findings of abnormal cognitive aging. There is some support for premature and accelerated cognitive aging among PLHIV in the existing literature especially among large and longitudinal studies and those with higher proportion of older samples. Future HIV and cognitive aging studies need to harmonize neuropsychological measurement methods and outcomes and use a large sample from collaborative multi-sites to generate more robust evidences.
\end{abstract}

Keywords HIV/AIDS · Aging $\cdot$ HAND $\cdot$ Neuropsychology $\cdot$ Systematic review

Electronic supplementary material The online version of this article (https://doi.org/10.1007/s10461-020-03053-3) contains supplementary material, which is available to authorized users.

Extended author information available on the last page of the article

\section{Introduction}

People living with HIV (PLHIV) are living longer than ever before with the widespread use of combination antiretroviral therapy (cART) [1,2]. The life expectancy of 
clinically stable PLHIV is approaching that of the non-HIV infected population [3], although this is hindered by those who have associated comorbidities [4]. As a result, the number and proportion of older PLHIV (over 50 years of age) are increasing [3, 5, 6]. UNAIDS [5] estimated that globally there were 5.8 million elderly PLHIV which account for $16 \%$ of the total PLHIV population, and it has increased to 7.9 million (21\%) in 2019 [7].

Previous studies in HIV and aging have found that HIV may lead to premature, accentuated and even accelerated aging [8]. Age-related conditions such as cardiovascular diseases (CVD), frailty, chronic renal disease and stroke were observed at a higher rate and at an earlier age among PLHIV than age-matched people without HIV even when accounting for lifestyle factors $[6,9,10]$. Immunosenescence, driven by chronic inflammation, chronic immune activation, and microbial translocation processes in chronic HIV infection, has been suggested as the underlying pathological process $[11,12]$.

As PLHIV are aging, neurocognitive health is becoming as important as physical and mental health as age is the primary risk factor for dementia [8] and HIV itself is a risk factor for neurocognitive impairment (NCI) known as HIV-associated neurocognitive disorder (HAND). According to Frascati criteria [13], HAND is classified into three stages: Asymptomatic Neurocognitive Impairment (ANI), Mild Neurocognitive Disorder (MND), and HIV-associated dementia (HAD), and requires the use of demographicallycorrected test scores as well as functional status and the exclusion of non-HIV causes of impairment. HAND, especially the ANI subtype still persists among PLHIV between 20-50\% even in the cART era [14-18].

HIV may also accentuate and/or accelerate brain aging directly through chronic neuroinflammation $[19,20]$ and indirectly by promoting premature and accentuated systemic aging and comorbid conditions such as CVD and kidney disease that are themselves associated with NCI [21]. Therefore, it is important to understand whether HIV also leads to abnormal neurocognitive and brain aging as with other agerelated conditions (i.e. whether HIV synergistically interacts with age to pose a greater risk for NCI or neurocognitive decline than the risk imposed by HIV or age itself). If this is true, PLHIV would systematically be at a much higher risk of dementia as they age, representing a major public health issue worldwide.

However, based on the literature, it is unclear whether HIV infection is associated with premature, accentuated and/ or accelerated neurocognitive aging or whether there is a negative synergistic effect of HIV and age on neurocognition [22-24]. To the best of our knowledge there has been no systematic review on this topic. A systematic review may lead to a higher level of evidence, and importantly aid in identifying which factors may be associated with neurocognitive aging in PLHIV assisting in future research directions.

In this review, we defined abnormal patterns of neurocognitive aging as follows: premature cognitive aging represents significant interaction effect of HIV status and age on cross-sectional neurocognitive test performance covering both the normal and abnormal performance range (i.e., HIV and older age synergistically lead to significantly poorer neurocognitive performance compared to HIV or/ and aging effect alone); accentuated cognitive aging represents significant interaction effect of HIV status and age on cross-sectional NCI rate, thus covering the abnormal performance range only (i.e., HIV and older age synergistically lead to much greater NCI rate compared to HIV or/and aging effect alone); accelerated cognitive aging represents significant interaction effect of HIV status and age on longitudinal neurocognitive test performance or incidence of NCI (i.e., HIV and older age synergistically lead to much steeper neurocognitive decline or significantly higher incidence of NCI compared to HIV or/and aging effect alone). Based on these definitions, it is possible that a cross-sectional study may only be able to detect premature aging and accentuated aging. A longitudinal study, though, may be able to detect not only premature and accentuated aging at baseline, but also accelerated aging at follow-up. However, these definitions necessitate the inclusion of an HIV-negative (HIV-) control group. Therefore, when an HIV-control group was not included, we were only able to determine the size of the aging effect within the HIV-positive (HIV +) participants, whether cross-sectionally or longitudinally, but not premature, accentuated or accelerated aging per se.

To achieve a comprehensive overview of the literature, we focused on the effect of aging on overall neurocognitive test performance or NCI rather than strictly following the HAND diagnosis criteria [13]. HAND is a diagnosis of exclusion that was conceptualized well before the aging effects of HIV were anticipated; and it is not possible to fully exclude non-HIV age-related conditions that may contribute or compound HIV-related NCI [24]. This strategy also ensures a more representative review because not all studies have correctly and comprehensively applied current standard diagnostic for HAND [25].

The overarching aim of this review was to synthesize and evaluate results derived from the existing literature in observational/interventional cross-sectional and longitudinal cohort studies conducted during the cART era in order to determine the extent of aging effects on neurocognition among PLHIV. More specifically, we first aimed at determining the magnitude of the age effect on the prevalence and incidence of NCI and overall neurocognitive test performance among PLHIV; and secondly, we aimed at determining the evidence for premature, accentuated and/or 
accelerated aging effects on NCI and overall neurocognitive test performance among PLHIV compared to HIV- controls.

\section{Methods}

\section{Search Strategy and Selection Criteria}

This review was carried out according to the Preferred Reporting Items for Systematic Reviews and Meta-Analyses (PRISMA) guideline [26]. A protocol for this review was registered on the PROSPERO website on 19/02/2019 (Registration ID—CRD42019123952). PubMed, EMBASE, CINAHL and PsycINFO databases were searched using the search terms presented in the Supplementary File 1 to find relevant literature, and this was conducted on 04/02/2019 (an example of the search is presented in the Supplementary File 1). Citation lists of the eligible articles from these databases were also manually searched for any additional relevant articles. A manual search was conducted again in Google Scholar on 20/12/2019 to find the articles published after the last database search.

The following inclusion/exclusion criteria were applied for the selection of studies. Studies were included if they analyzed and reported the effect of age on neurocognition among PLHIV as one of their major findings, if they were conducted in the cART era typically after 1996, if they included only adult participants (aged $>18$ years), and if they included PLHIV of whom $>30 \%$ were on cART. Studies were excluded if they were not published in a peer review journal, if they were not written in English, and if the sample size was $<30$.

Articles were initially screened from reading titles and abstracts by HLA. After this initial screening process, fulltext articles of all the relevant articles were obtained. The full texts were then reviewed by HLA and independently by MA to assess eligibility criteria. A consensus discussion was conducted with LC when eligibility was uncertain. Final selection of the articles was made by matching the articles chosen between two reviewers.

Data were extracted by both HLA and MA using a Microsoft Excel format developed by HLA which covered the following areas: Study Characteristics, Study Method and Results (see the Supplementary File 2 for the detailed areas assessed). Data collected by two reviewers were compared; and any mismatched information was discussed again with $\mathrm{LC}$ to form a final consensus.

\section{Data Analysis}

Critical appraisal tools from Joanna Briggs Institute (JBI) [27] for analytical cross-sectional studies and cohort studies were used to review the quality of the eligible articles.
The JBI tools were adjusted to be more relevant to the topic of this review. Specifically, we first specified whether the study included a demographically comparable HIV- control group and/or used demographically corrected cognitive scores. Further, we adapted the rating classification across the JBI tools from "Yes, No, and Not Clear" to "Yes, Partly, No, and Not Applicable" to better rate the neuropsychology methodology of each study. The detailed definitions of all the items assessed are presented in the Supplementary File 3. The quality assessment was conducted independently by both HLA and MA. Any discrepancy on the quality ratings between the reviewers was discussed with $\mathrm{LC}$ to arrive at a final consensus. No study was dismissed a priori as the systematic review intended to provide a transparent snapshot of the quality of the cognitive aging literature in HIV at this moment in time.

A meta-analysis could not be conducted as initially intended because study designs, methods and choice of neuropsychological measurement methods and outcome variables were too heterogeneous among studies. We therefore used a narrative synthesis approach [28] to integrate the review findings. When synthesizing and comparing results across studies, we presented some figures with some quantitative outcomes, but they only represent descriptive aspects of studies. Outcomes from the studies were synthesized separating studies with and without an HIV- control group. From the studies with HIV-controls, the presence and magnitude of premature and accentuated (from cross-sectional studies) or accelerated aging effects (from longitudinal studies) were interpreted by evaluating the presence of an interaction effect between HIV and age on neurocognition. In studies without an HIV- control group, we were able to only identify whether there was a significant aging effect on the level of neurocognitive test performance or the prevalence and incidence of NCI/HAND within the HIV + participants. We also extracted and presented the range of effect size of aging effect on neurocognition among the studies without HIV- controls. However, studies which did not use demographically corrected scores were not included in the reporting of effect sizes. This was done to present the best interpretable data regarding the effect of chronological age in PLHIV versus normal aging.

\section{Results}

The database search process returned a total of 436 articles. After screening, 37 articles ( 31 cross-sectional and 6 longitudinal) were selected (see the PRISMA Flow Chart in Fig. 1 for detailed screening process). Studies' characteristics are presented in Table 1 for studies that included an HIV- control group and Table 2 for studies that did not include a control group, whereas the methods and outcomes of the 


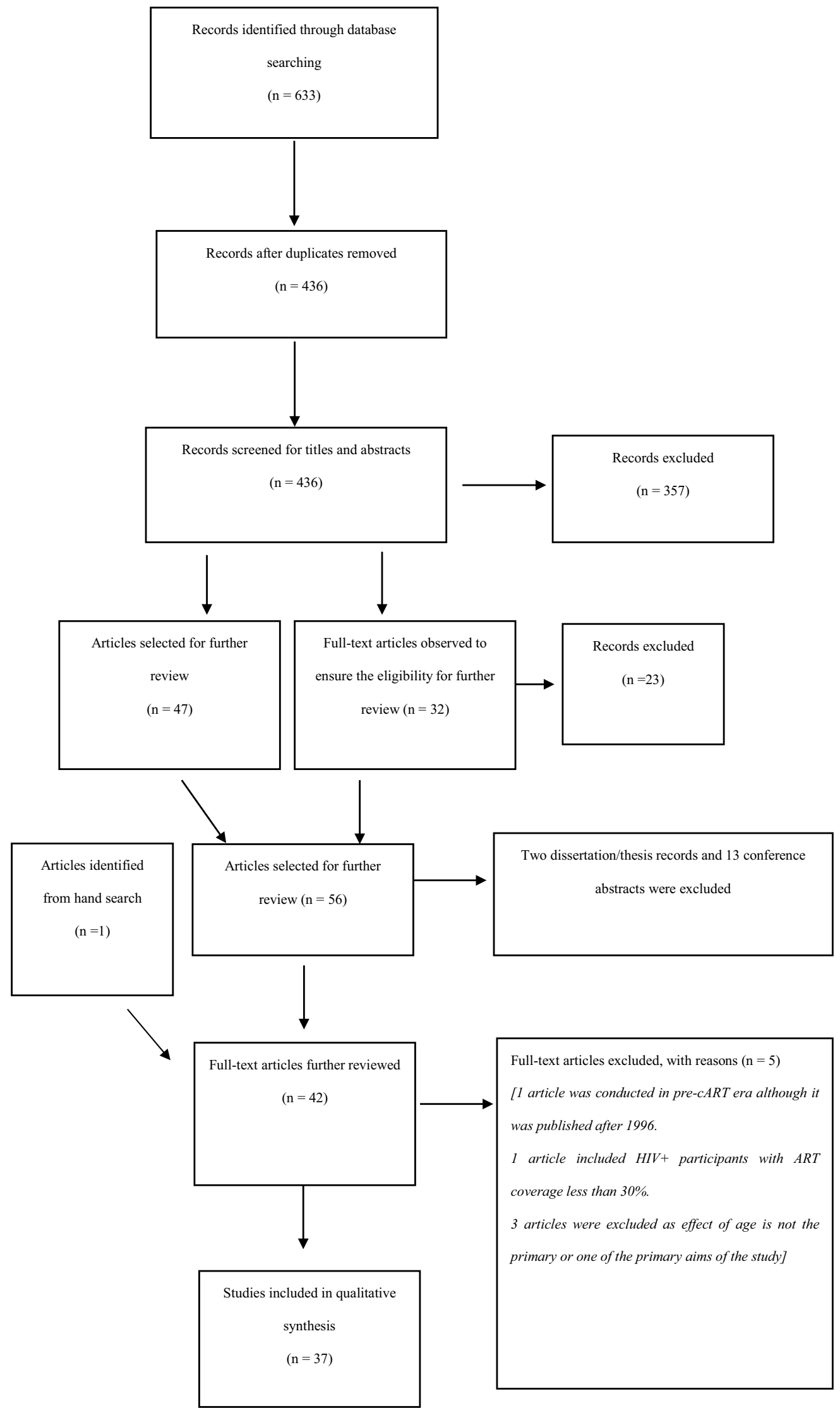

Fig. 1 CONSORT Flow diagram for literature searching 


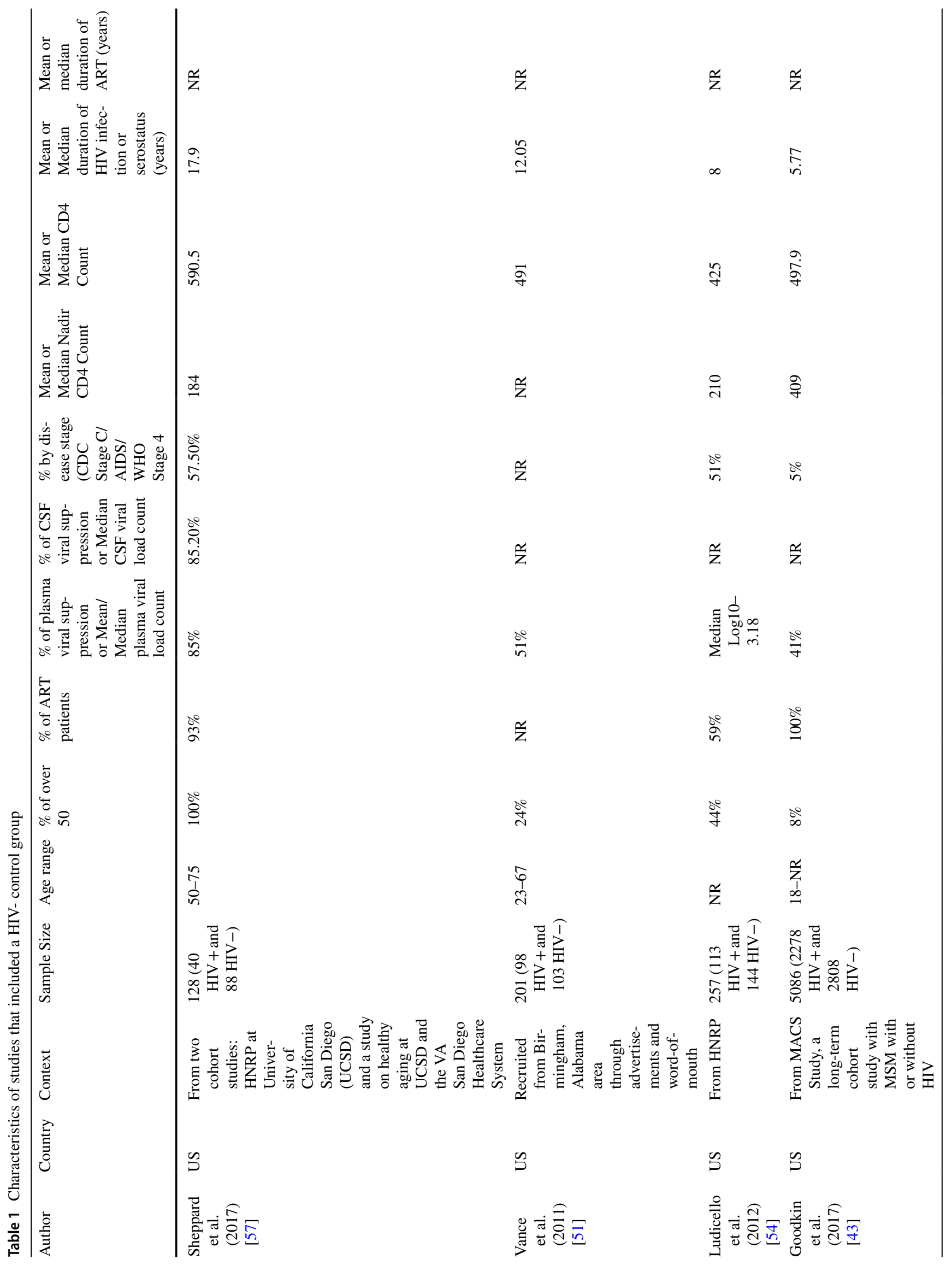




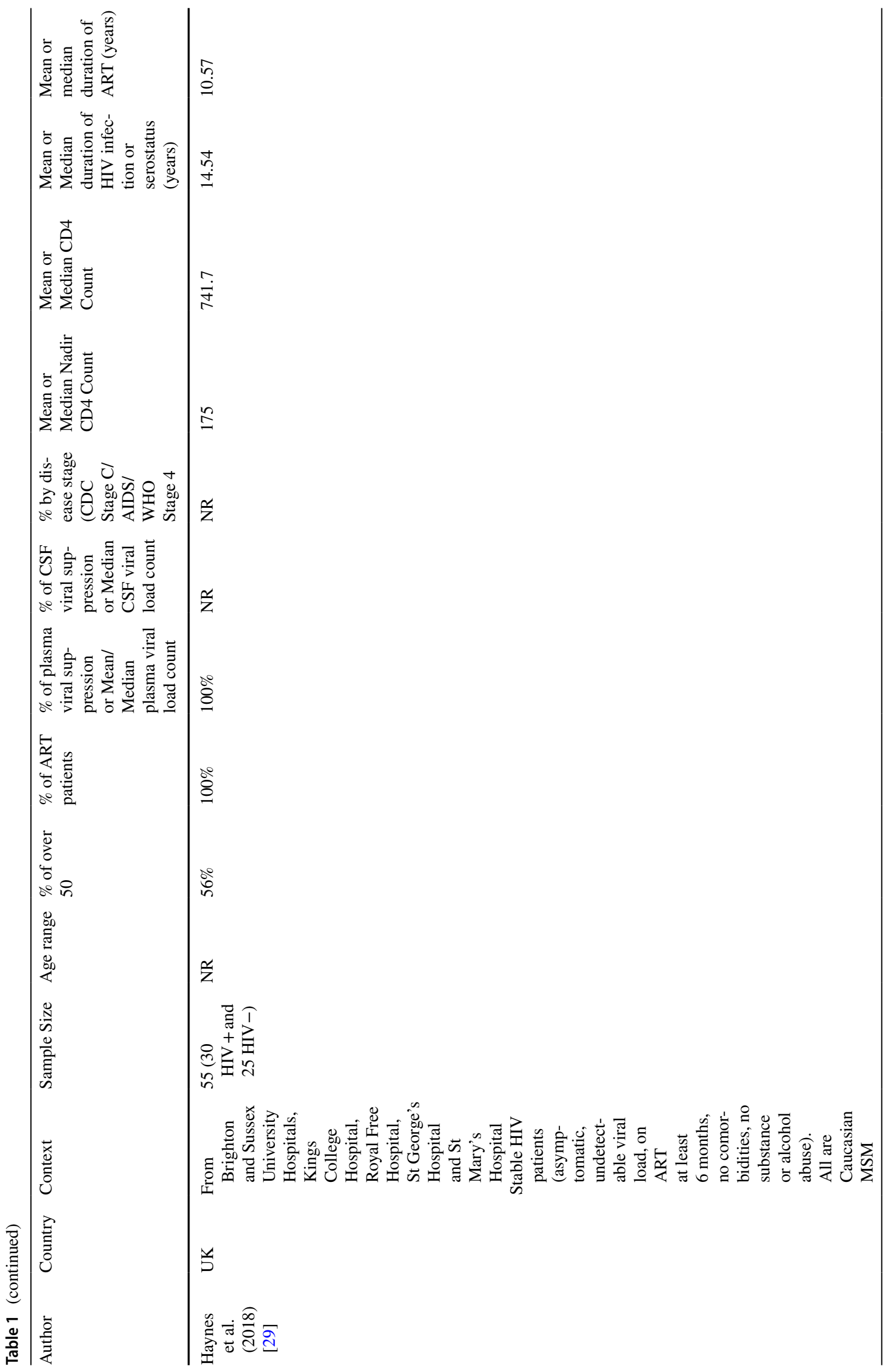




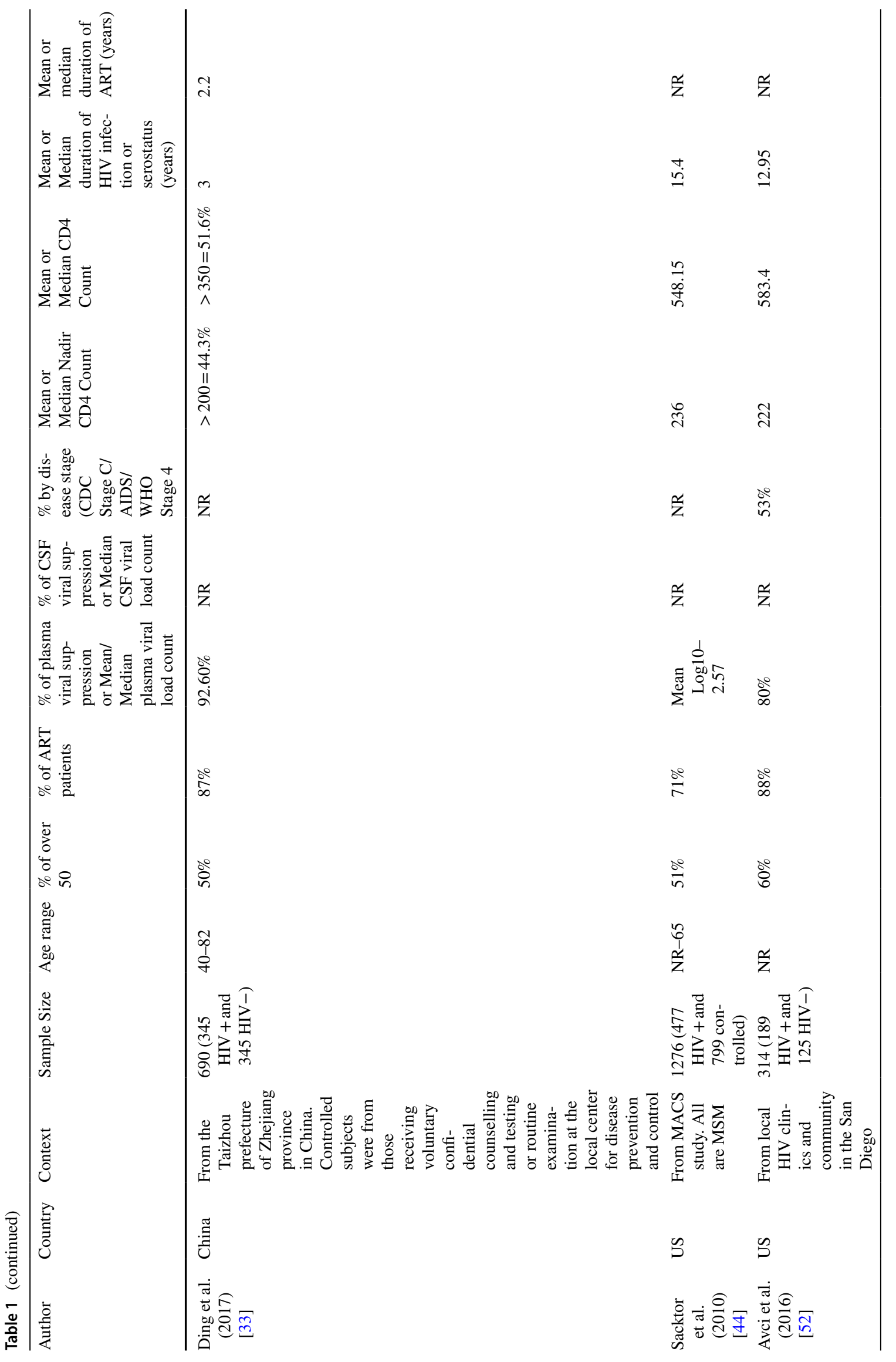




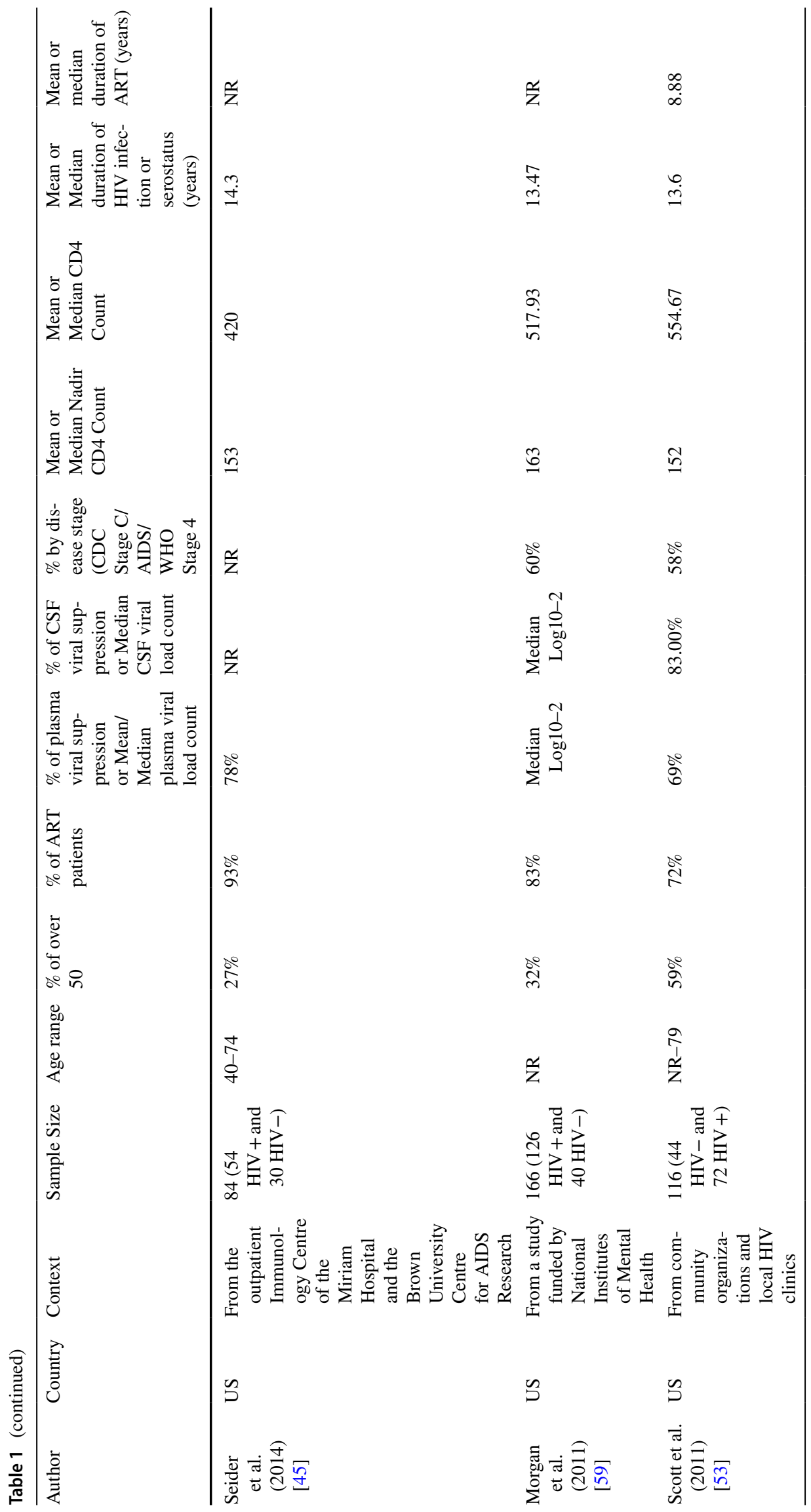




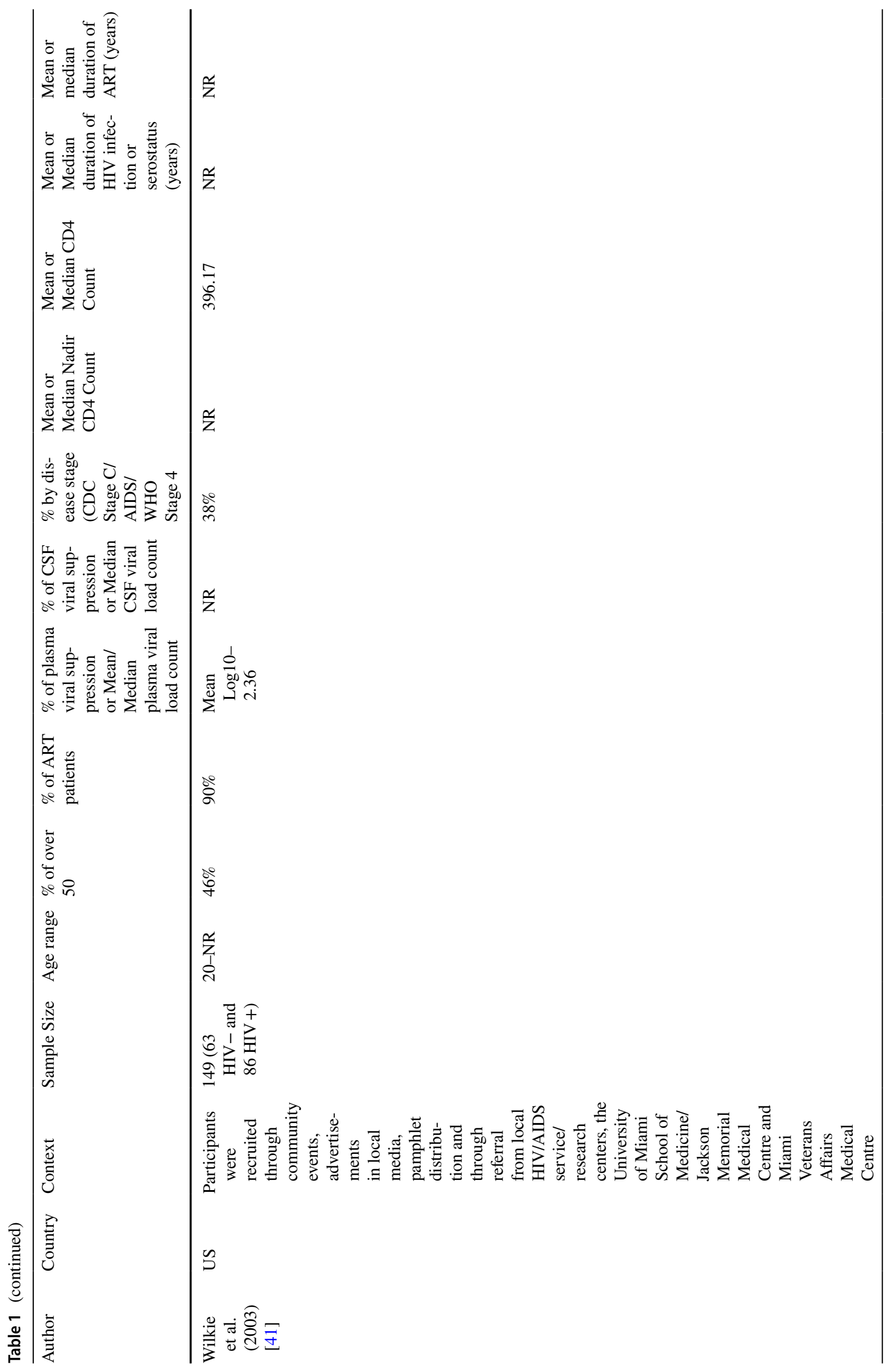




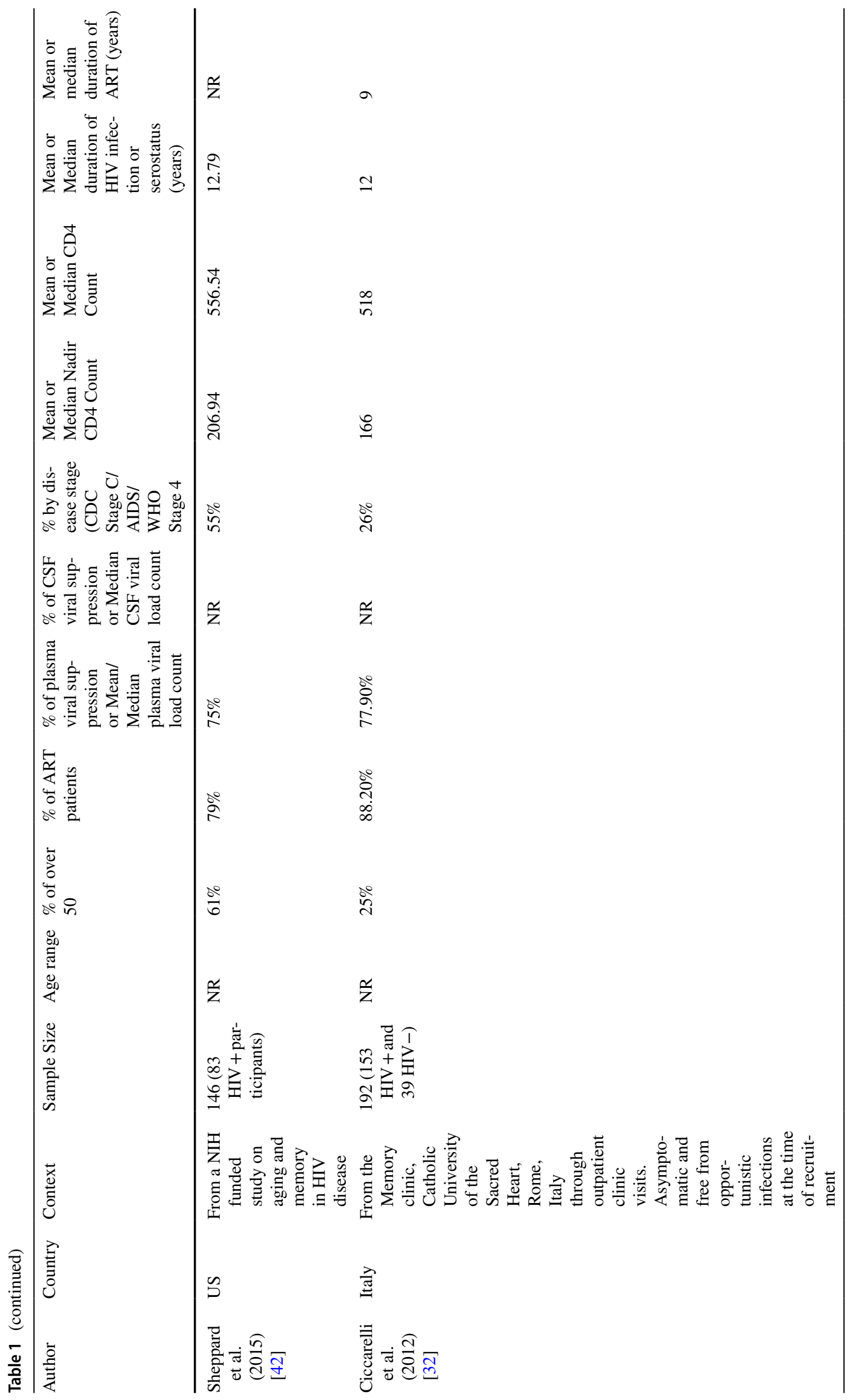




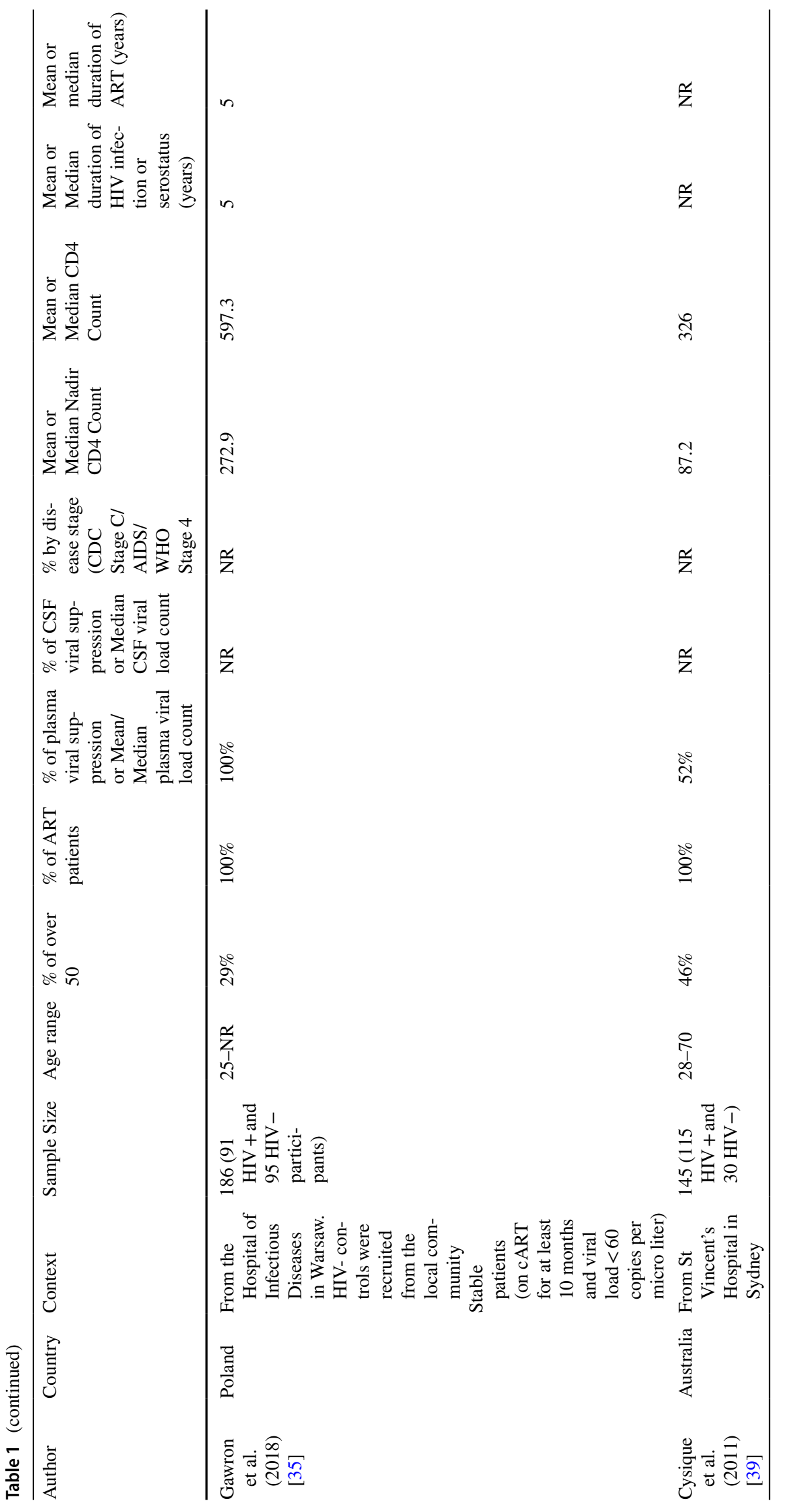




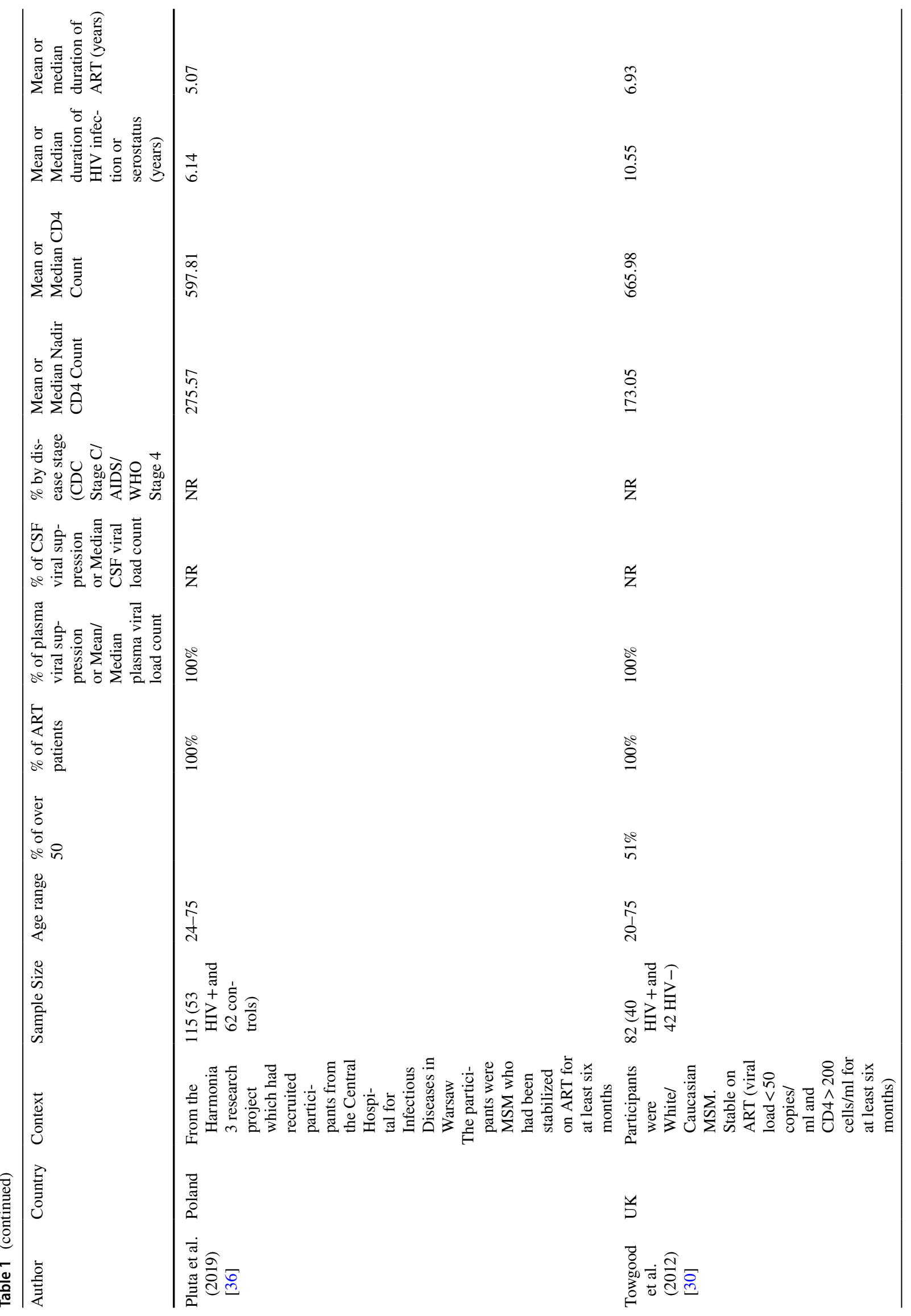




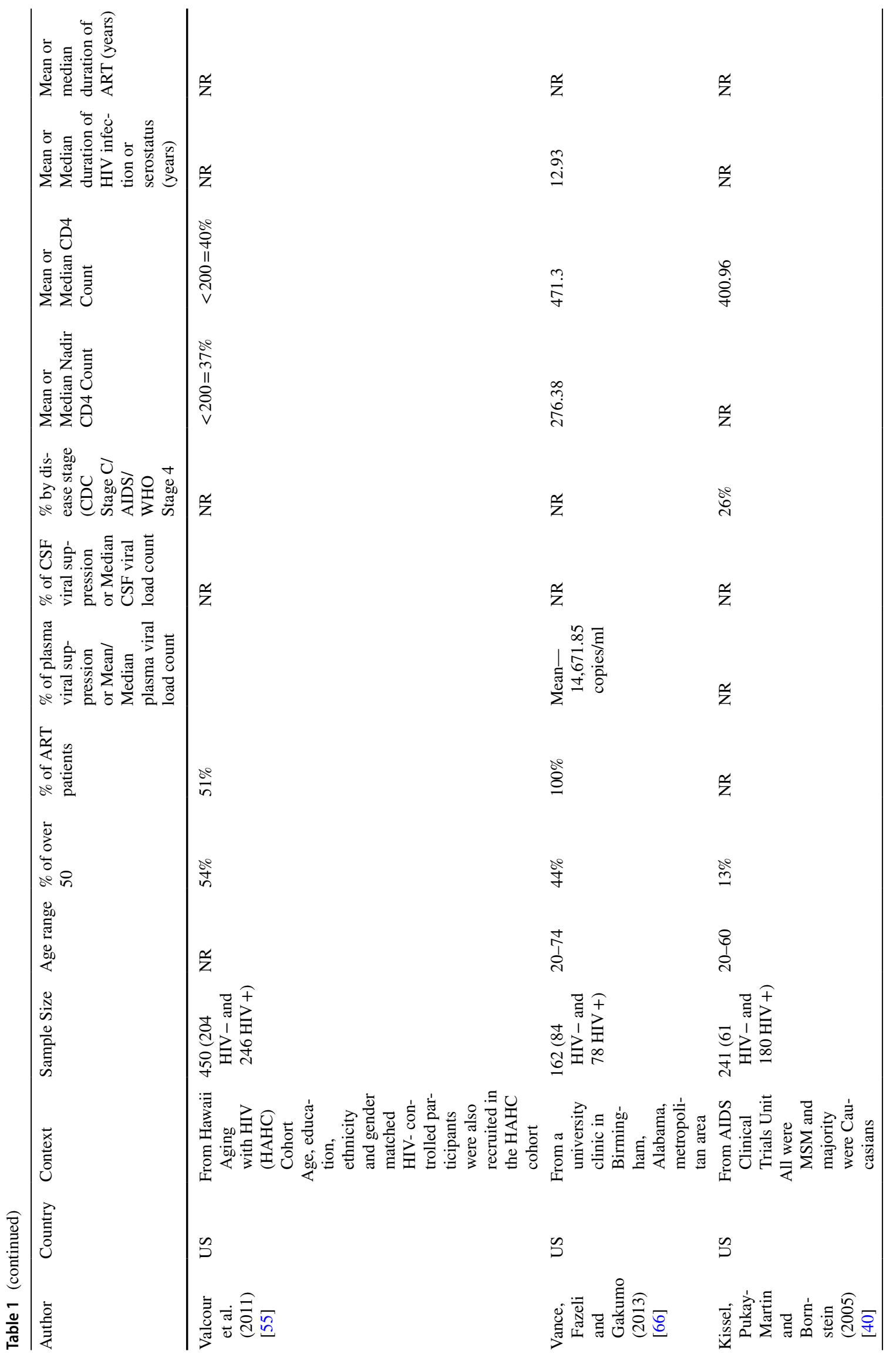




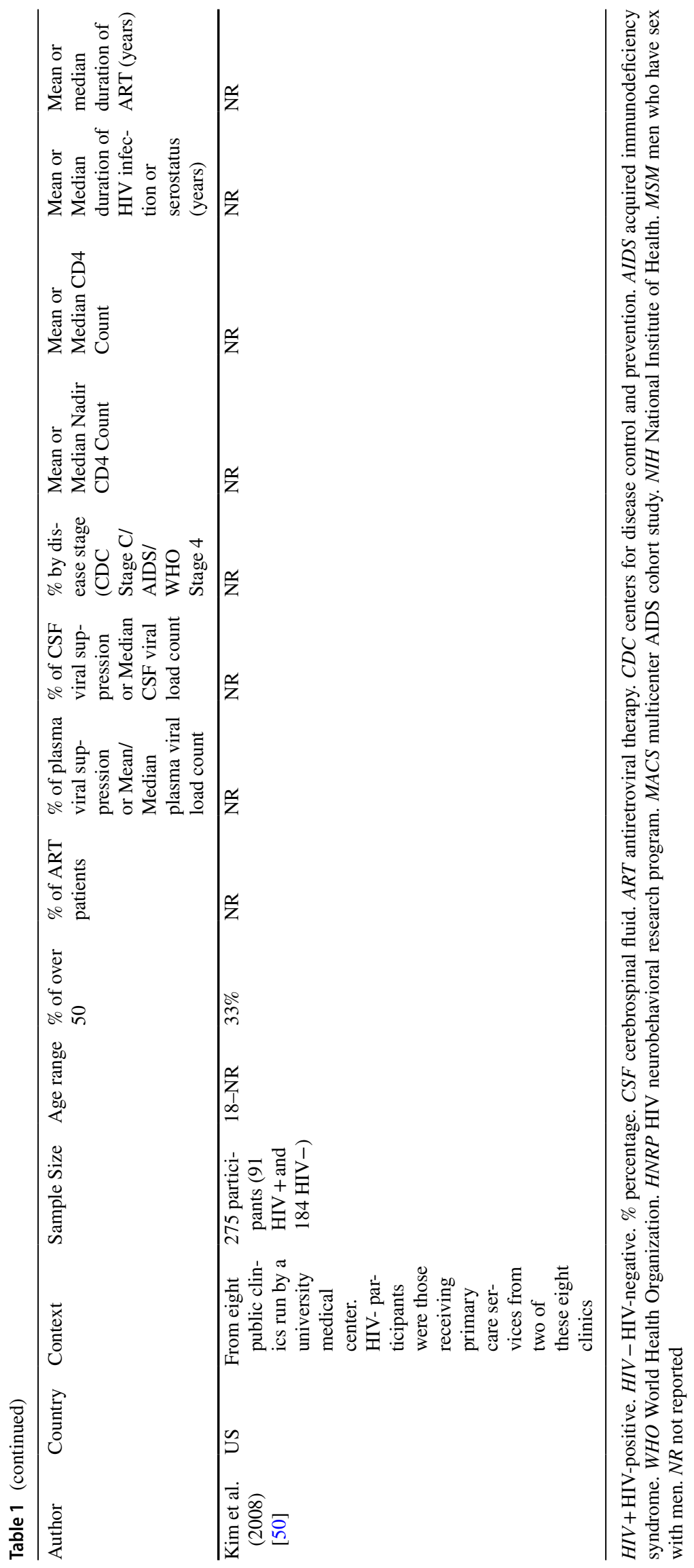


studies are detailed in Table 3 (studies with an HIV- control group) and Table 4 (studies without a HIV- control group).

\section{Study Characteristics}

\section{Locations and age of studies}

The majority of the studies were conducted in the US while two studies originated from the UK [29, 30], Italy [31, 32], China [33, 34], and Poland [35, 36], and one was carried out in Japan [37], Brazil [38], and Australia [39]. No study was conducted in low-income countries. Over two thirds of the studies (28 studies) were published in the last decade (2011-2019). The oldest record included was published in 2003 [40, 41].

\section{Study design}

In terms of the study design, only 6 out of 37 studies were designed longitudinally [29, 31, 42-45]. None of the 37 studies were interventional. The follow-up periods among these six longitudinal studies were one-year follow-up in three studies, and 5 years, 4.2 years (average) and 4.7 years (average) respectively in the other three studies respectively.

Age was the only primary predictor in all studies, but in eight studies, age was investigated as a co-primary exposure along with a HIV-related variable such as duration of HIV infection [37] or a non-HIV related factor such as education [46] and alcohol use [47]. Neurocognition was also the sole primary outcome of interest in all but five studies [29, 30, $35,36,48]$, which also identified the effect of age on changes in neuroimaging.

\section{Sample size}

The majority of the studies included samples lower than $300(29 / 37)$ and the median sample size across the studies was 192 (IQR: 116-267). Five studies [33, 37, 43, 44, 49] recruited more than 500 participants. Two multi-site studies included very large samples: one with 3313 participants [49] and another one with 5086 samples [43].

\section{Inclusion of HIV- controls}

Fifteen studies did not include HIV- controls. In studies that included a HIV-control group, the proportion of HIV- participants among total samples varied from 20-69\% ( $\geq 50 \%$ were controls in $48 \%$ of studies). Compared to HIVcontrols, HIV + participants were younger in four studies $[43-45,50]$ and older in two studies $[29,51]$. There were also differences in other demographic factors such as education and ethnicity between HIV + and HIV- participants in
11 studies [40-45, 50-53], and they controlled these factors in the analyses.

\section{Samples ascertainment, gender, and ethnicity}

In 17 of 36 studies, participants were recruited from existing research projects, such as the HIV Neurobehavioral Research Program (HNRP), Hawaii Aging Cohort Study and Multicenter AIDS Cohort Study (MACS). In the remaining studies, participants were recruited from HIV treatment clinics or the community. Twenty eight studies excluded participants with a major confounding condition for HAND as delineated using in the Frascati Criteria [13] such as major neurological and psychiatric disorders, current substance abuse and history of head trauma with loss of consciousness more than $30 \mathrm{~min}$. Six studies [29, 30, 36, 40, 43, 44] included men who have sex with men (MSM) participants only, and three $[29,30,40]$ of these recruited only Caucasian participants. Two studies $[35,46]$ included only male participants. Two British studies [29, 30] and two Polish studies $[35,36]$ enrolled solely virally suppressed HIV + participants (plasma HIVRNA $<50 \mathrm{cp} / \mathrm{mL}$ ). One study [31] included only formally diagnosed HAND cases and compared the distribution of young and old age groups among mild and severe cases of HAND.

\section{Samples' HIV disease characteristics}

Major disease markers such as duration since HIV diagnosis, nadir/current $\mathrm{CD} 4+\mathrm{T}$ cell count, duration of ART and viral control level were also presented across studies, although there was heterogeneity in how the information was presented and how much detail was provided. In one quarter of the studies, all HIV + participants were on ART. The percentage of patients on ART was lower than $70 \%$ in four of the studies [31, 54-56], all published before 2013. In 13 studies, the proportion of HIV + participants on ART was compared between the younger and older groups. A higher proportion of older HIV + participants was reported to be on ART across studies.

Standard HIV disease staging was reported in 15 studies. The proportion of WHO Stage 4 or CDC Stage C varied between 5-73\% among studies; and in eight studies more than $50 \%$ of HIV + participants had been diagnosed with WHO Stage 4 or CDC Stage $C$ in eight studies.

Nadir CD4 + T cell count was reported in 27 studies, while 34 studies gave the current $\mathrm{CD} 4+\mathrm{T}$ cell count among their HIV + participants. The mean or median nadir CD4+ T cell count was lower than 200 in 10 studies, and the current CD4 + T cell was higher than 500 among participants in 14 studies. The current $\mathrm{CD} 4+\mathrm{T}$ cell count was differentiated between the younger and older groups in 23 studies, and the younger group had a higher level in 13 of the studies. 


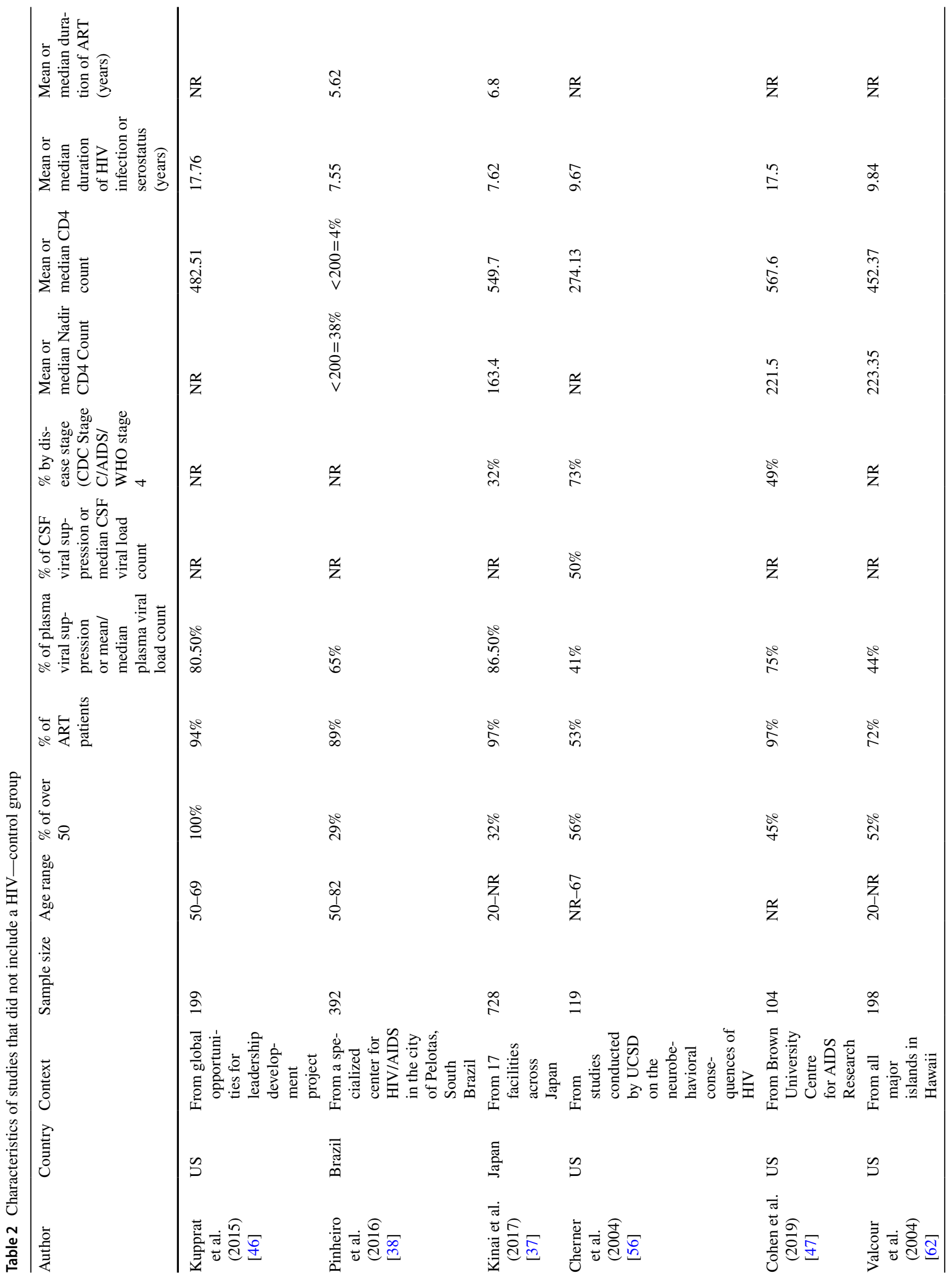




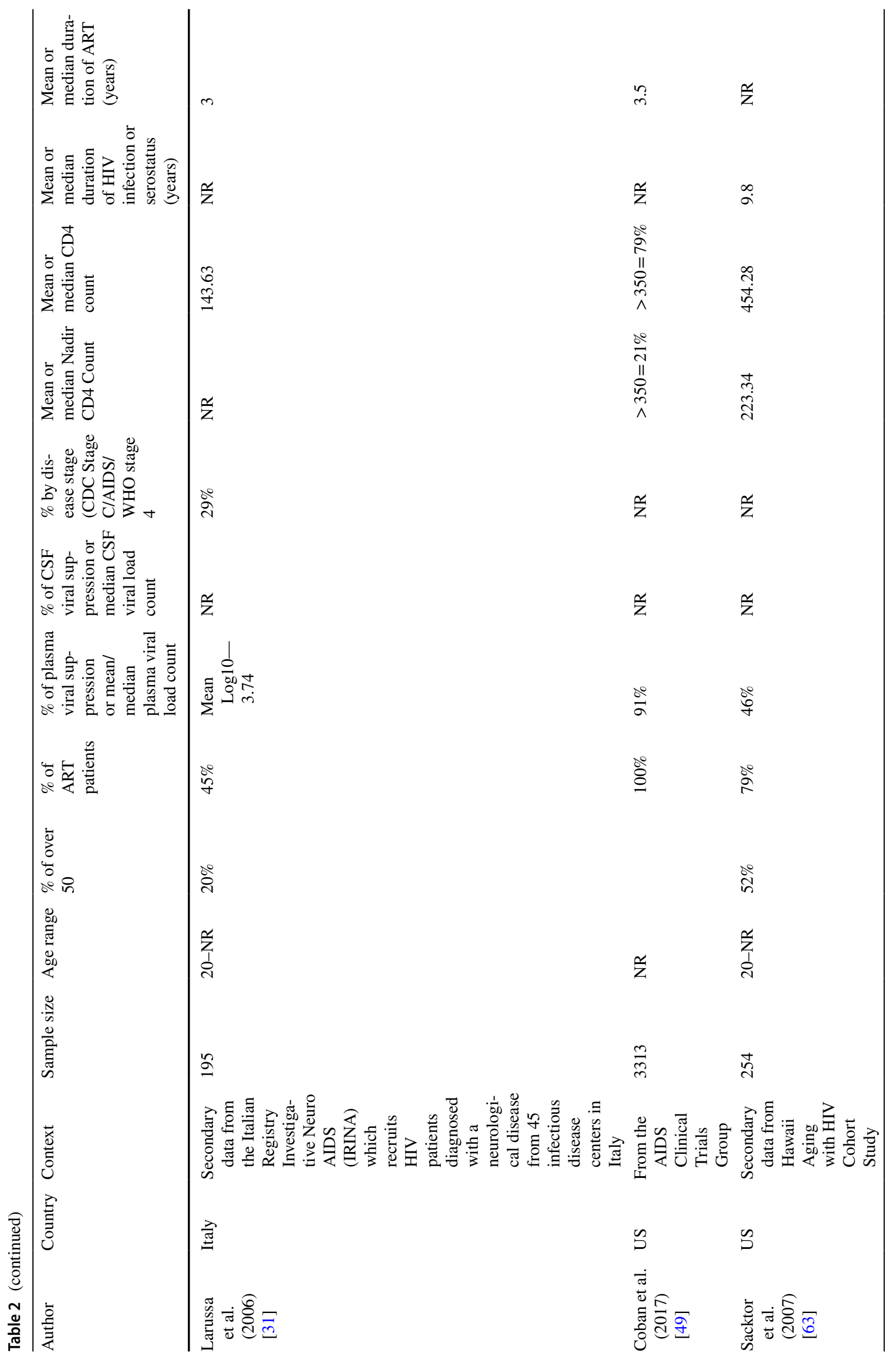




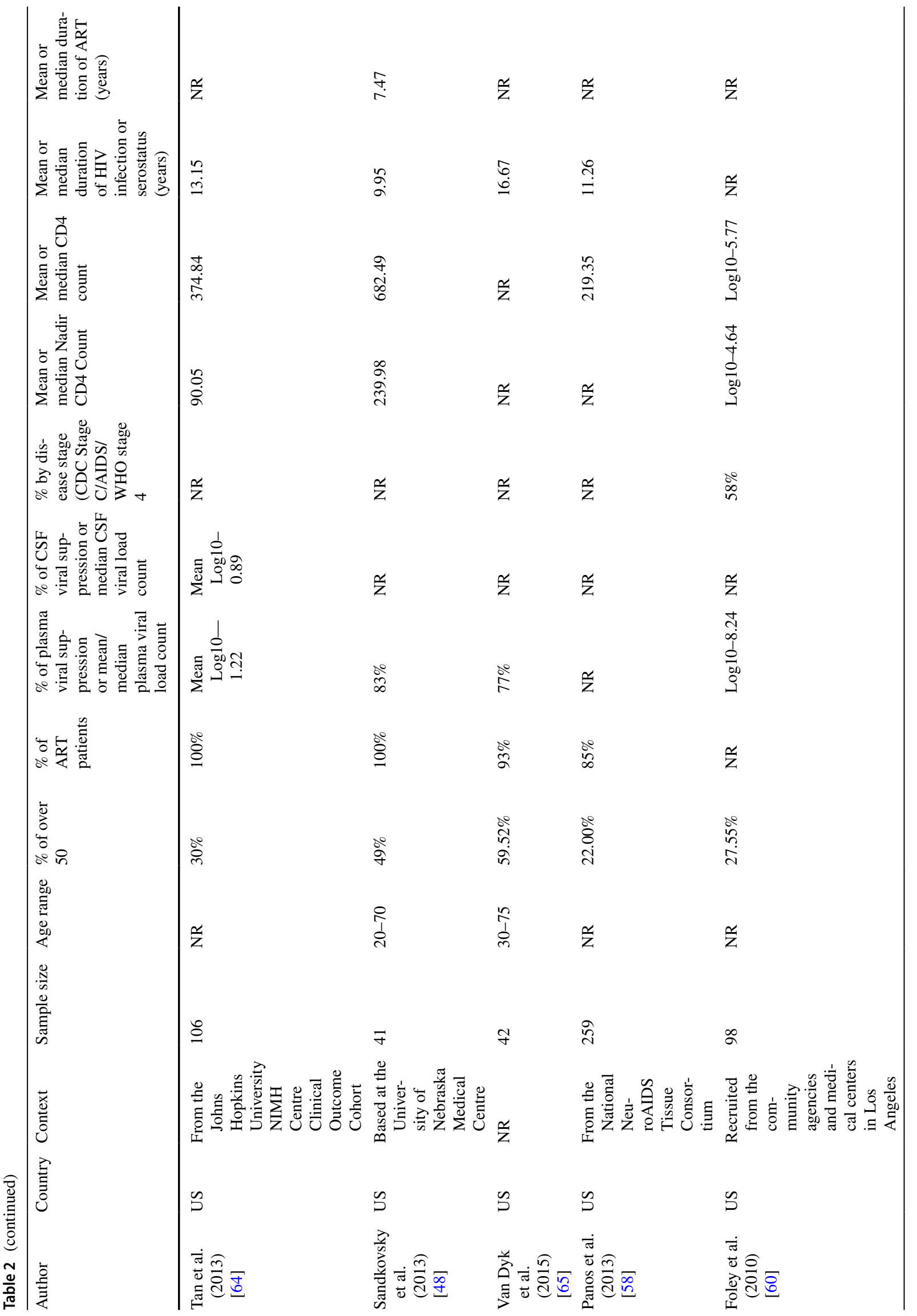




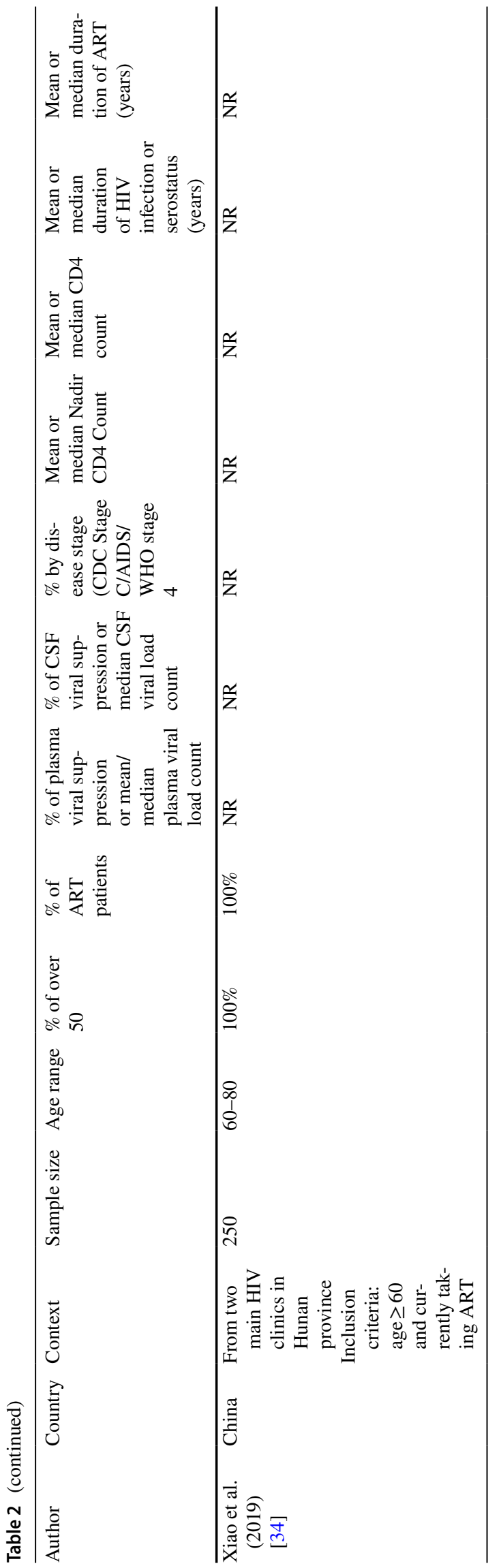

Nineteen studies compared the nadir CD4 + T cell count level between the age groups as well, and a higher proportion (15/19) of studies showed that the older participants had lower nadir CD4 + T cell counts.

Plasma viral load information was reported in 33 of 37 studies. The percentage with plasma viral suppression was reported in 26 of 36 studies. In 17 of 26 studies, $\geq 75 \%$ of participants had achieved plasma viral suppression. When the percentage of participants with plasma viral suppression was compared between young and old groups, it was higher among the older groups in 10 of 13 studies which assessed this question. Cerebrospinal fluid (CSF) viral load was reported in only five studies. Among them, the percentage of participants with CSF viral control was reported in three studies (50\% in one study [56] and $>80 \%$ in another two studies [53, 57]).

Twenty-eight studies reported the mean or median duration of HIV infection or serostatus (range 3-18 years). In 17 of 28 studies, HIV duration was greater than 10 years. Mean or median duration of ART was stated only in 12 studies and varied from 2-11 years.

\section{Reporting of comorbidities}

Other known contributing factors to neuropsychological performance were collected across studies, but again with some heterogeneity in the level of detail. Mood status (anxiety or/and depression) was only assessed in 26 of 37 studies. Alcohol or substance use was reported in 18 of 37 studies. Co-infection with HCV or HBV was also reported in 14 of 37 studies. Information on age-related comorbidities such as hypertension (10/37 studies), diabetes (9/36) and CVD events (4/37) were scantly reported.

Across studies, findings indicated that HIV + participants compared to the HIV- controls tended to have higher prevalence of anxiety, depression and substance abuse. Further, across studies, older participants were more likely to have age-related comorbidities such as hypertension and were less likely to be a current substance user.

\section{How was age defined?}

The age of the youngest person included across the studies was 18 years old and the oldest person included was 82 years old. All but three studies investigated the effect of age across the adult life span. The three studies examined the effects of age only among participants over 50 years of age [34, 46, 57]. Age was categorized in 27 studies for major analysis and nine studies analyzed age as a continuous variable [34-36, 39, 43, 49, 58-60]. In most instances, age was categorized into two groups while four studies [31, 33, $44,46]$ divided age into more than two groups. The lower cut-off point for the older group was 50 years of age in all 


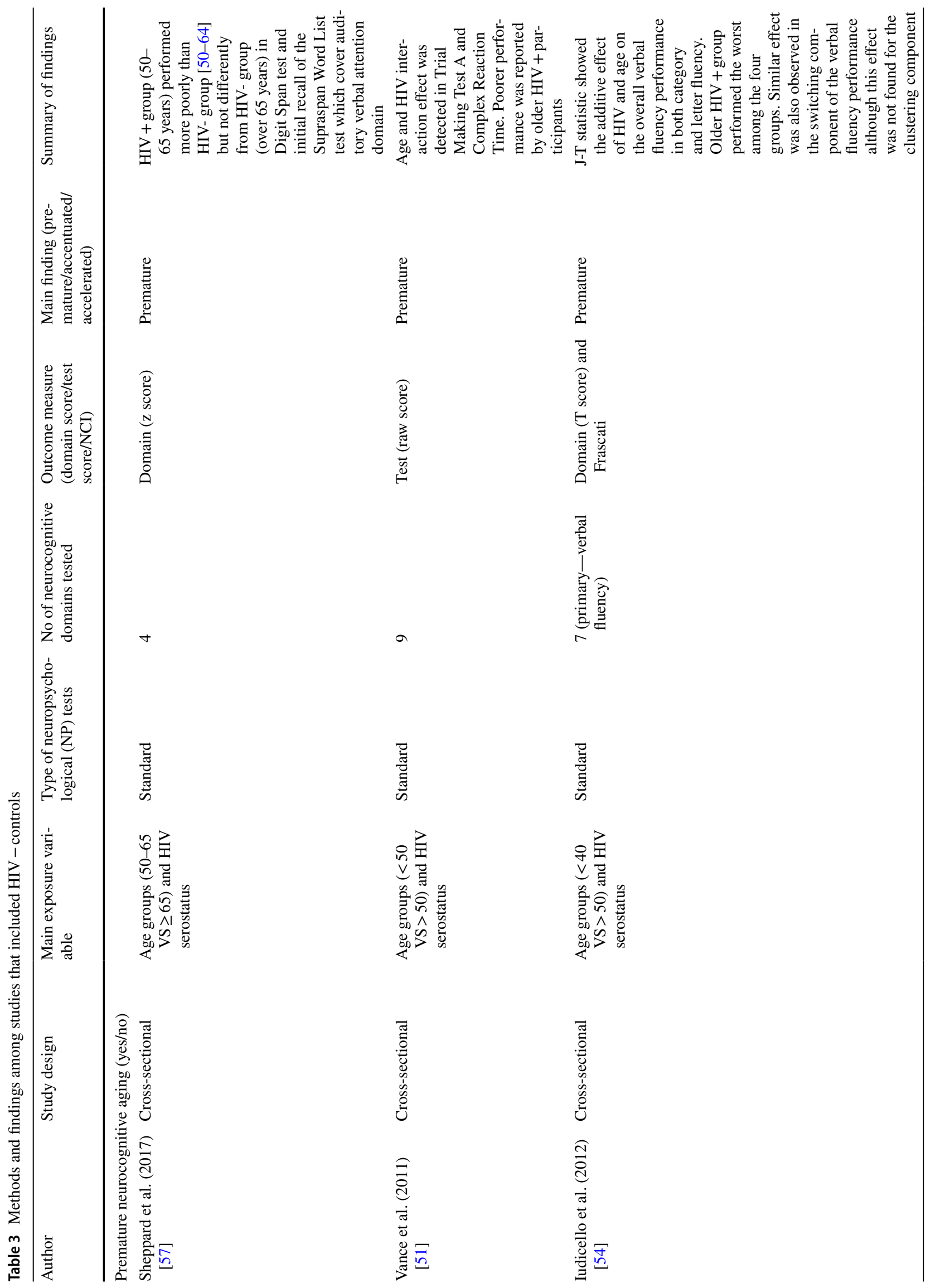




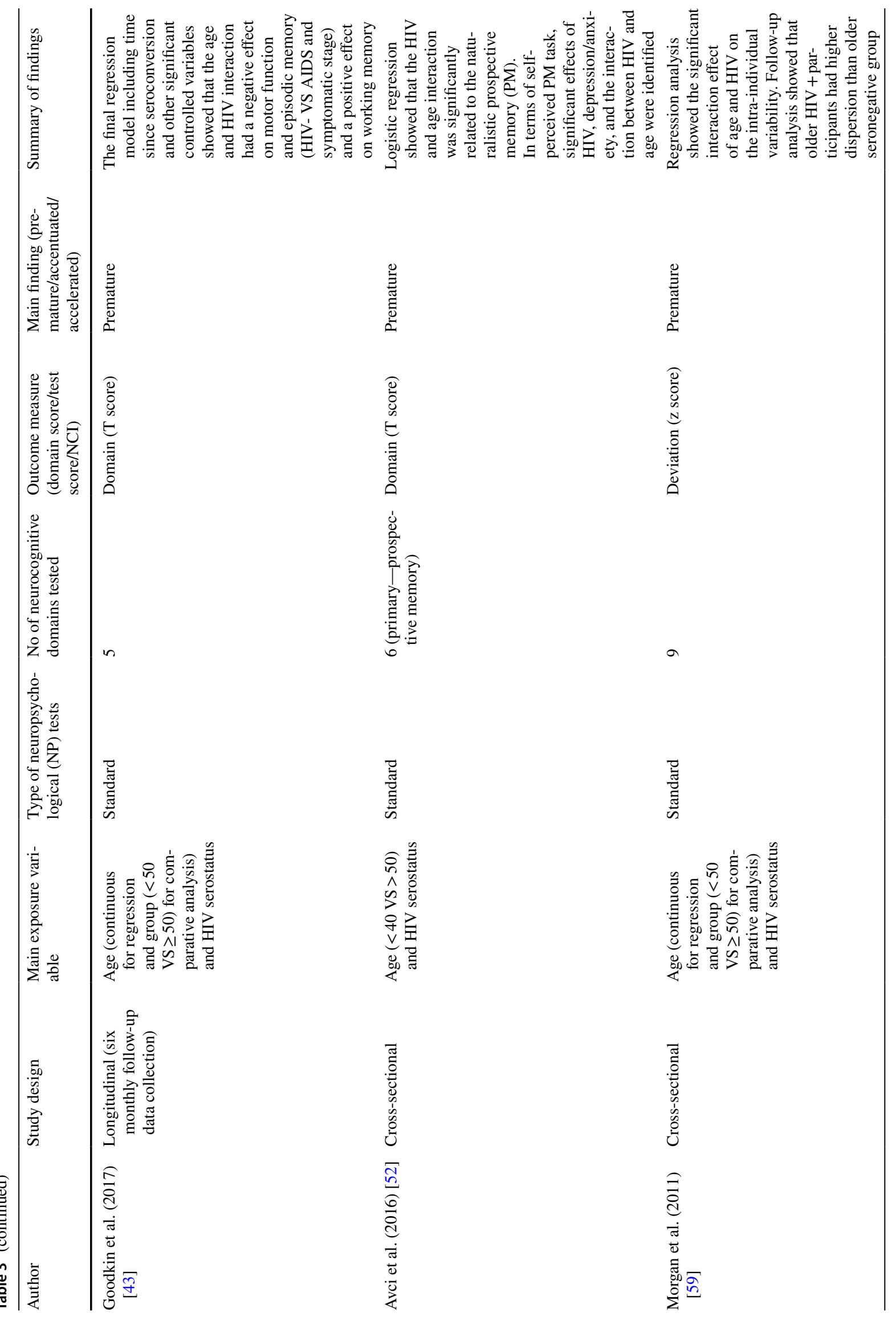




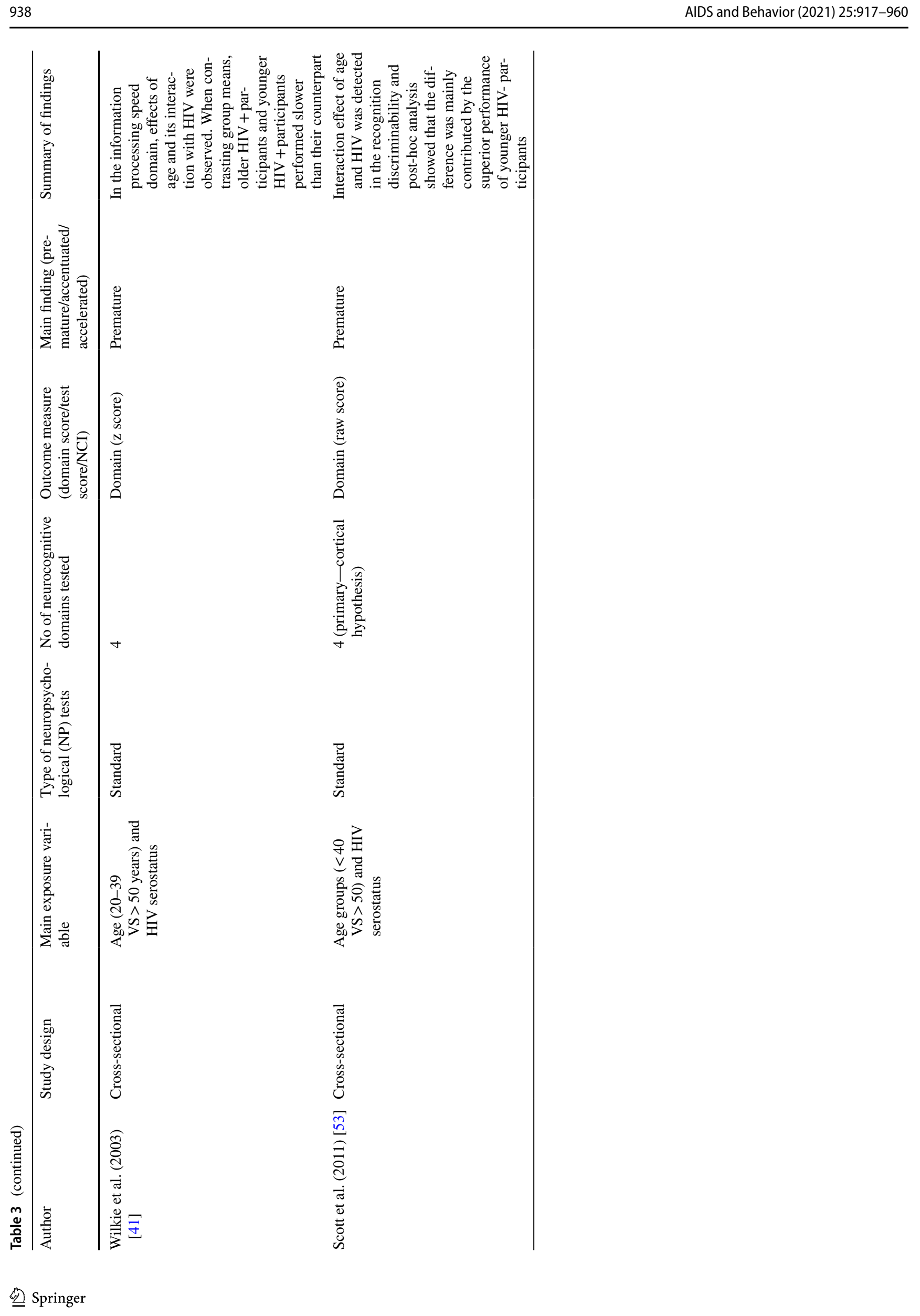




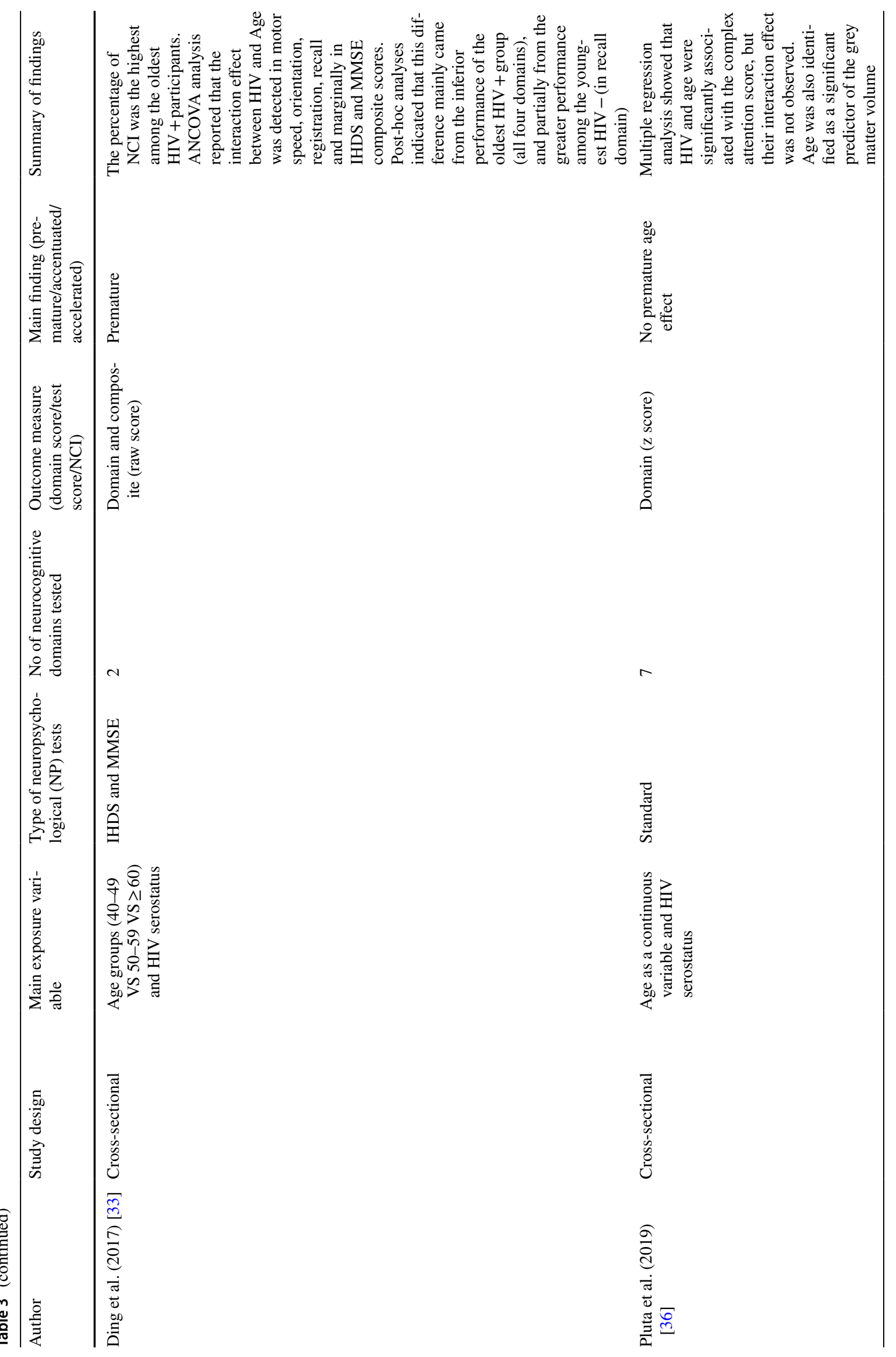




$$
\Gamma
$$




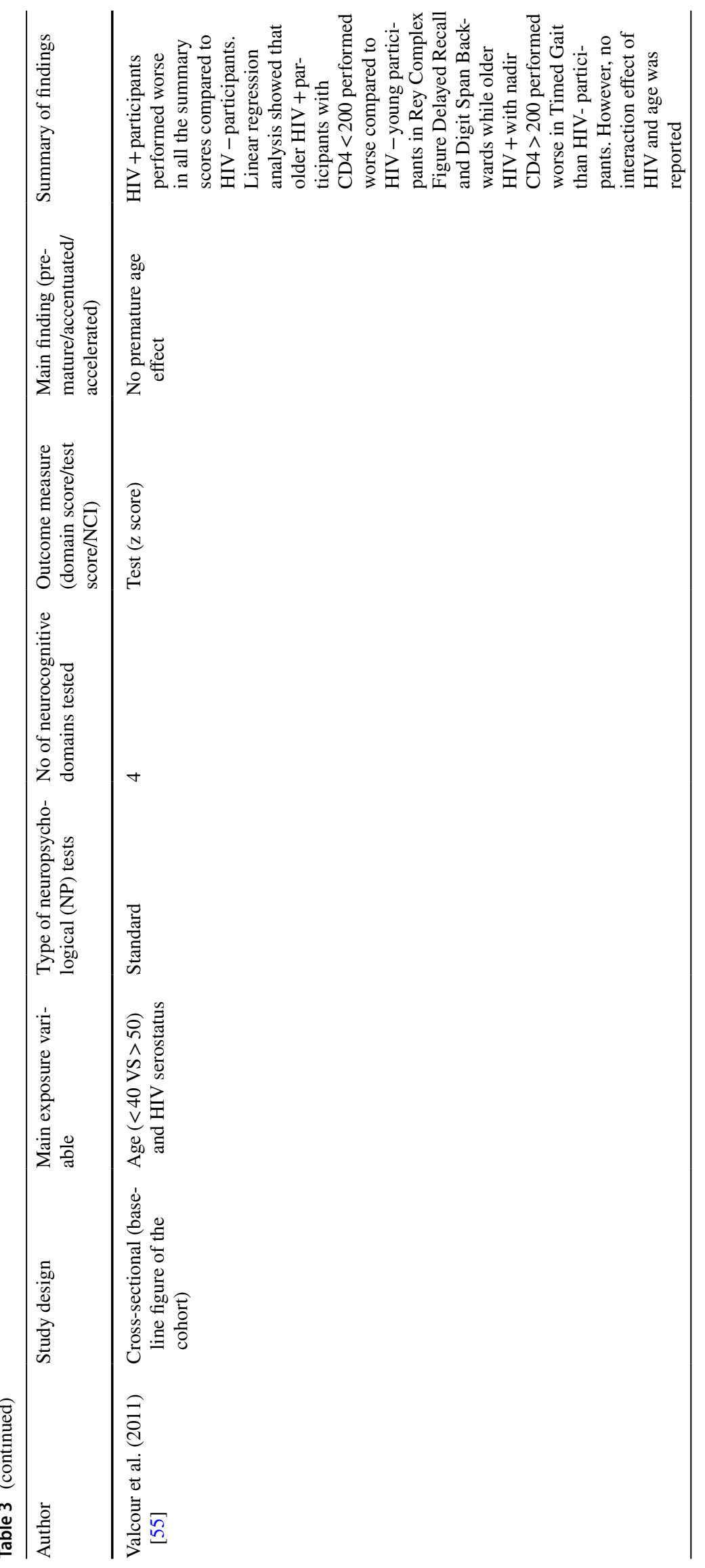




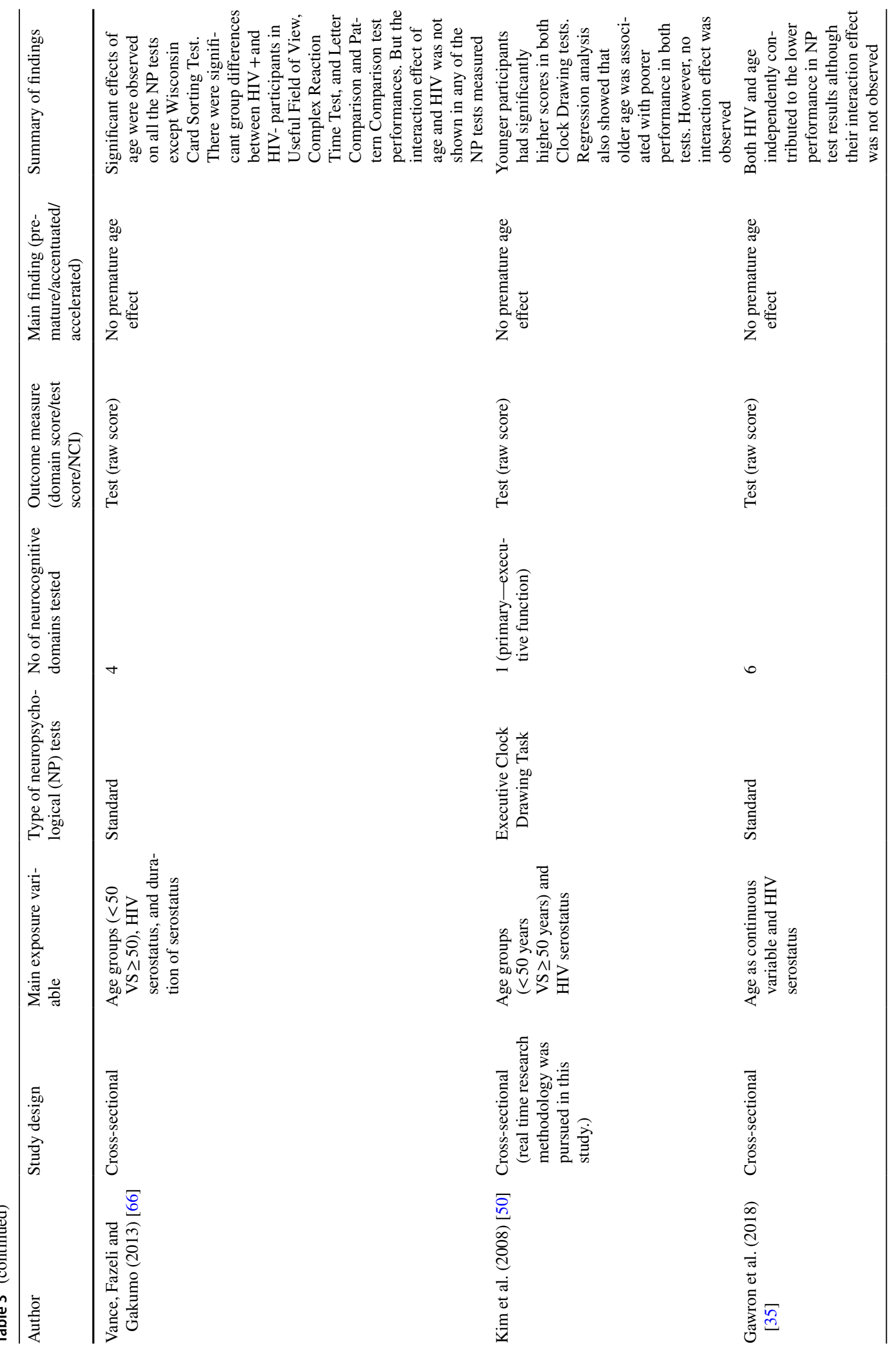




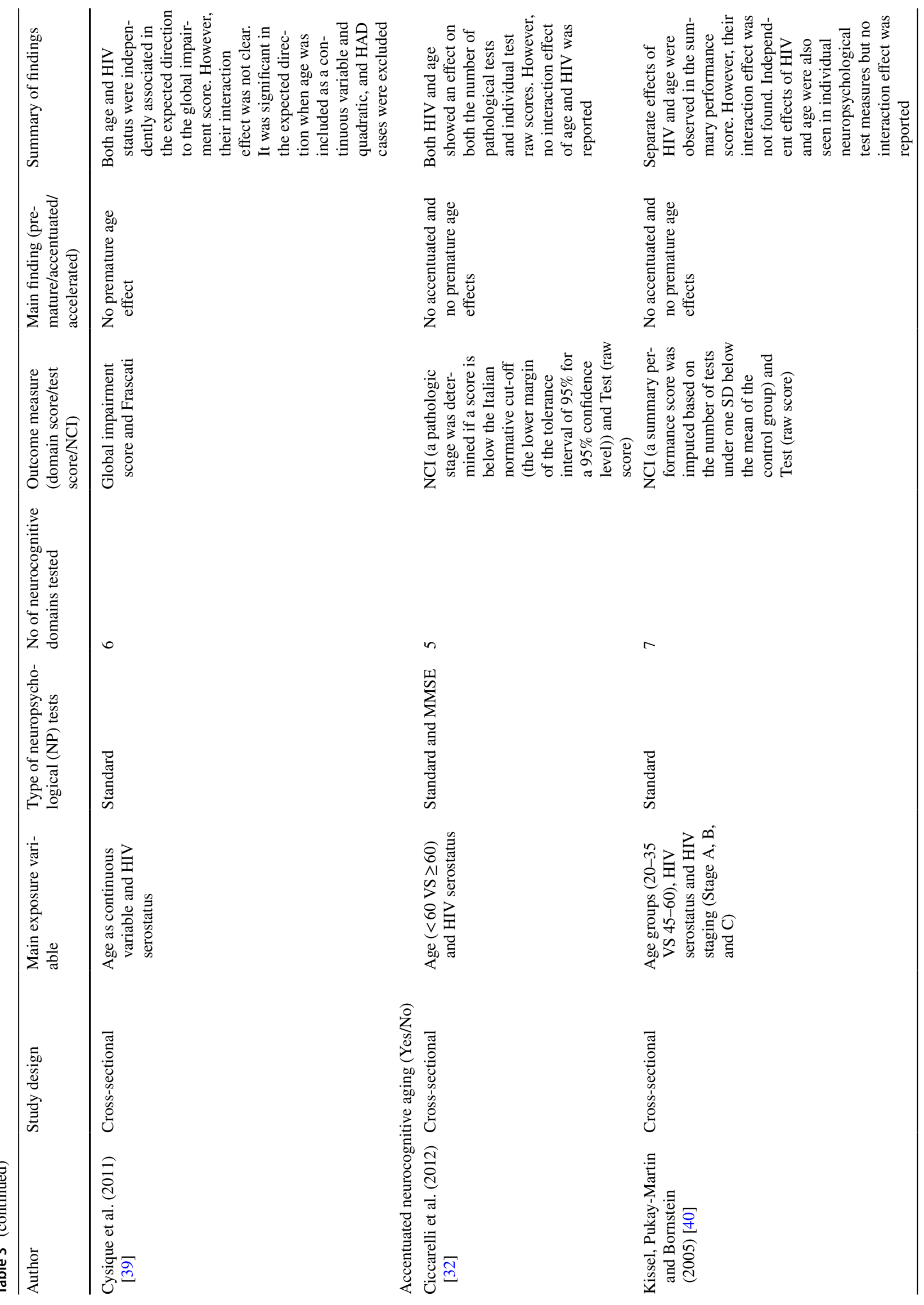




\begin{tabular}{|c|c|c|c|}
\hline 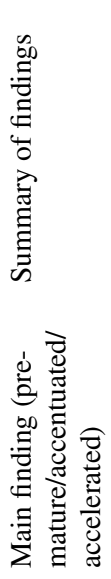 & 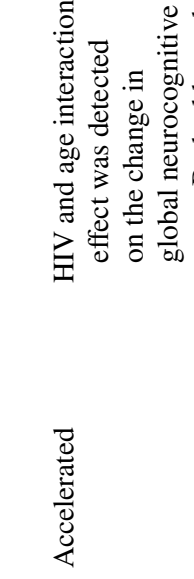 & 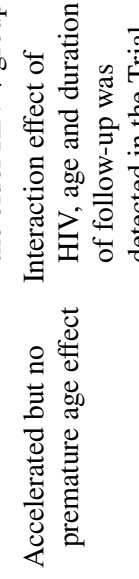 & 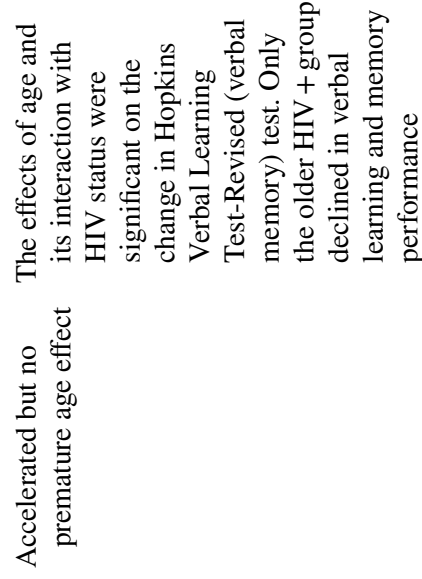 \\
\hline 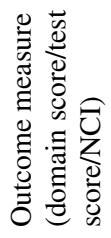 & 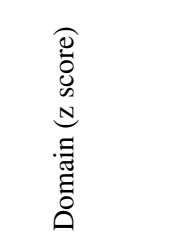 & 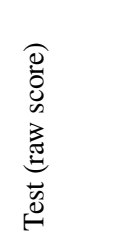 & 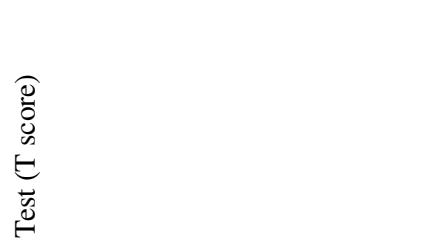 \\
\hline 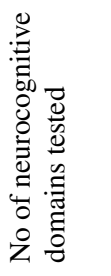 & $n$ & 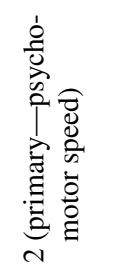 & 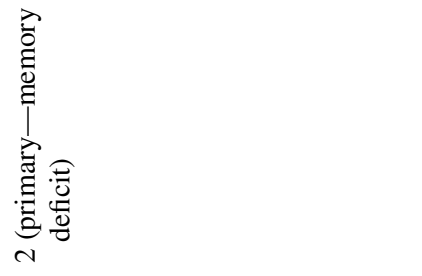 \\
\hline 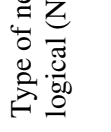 & 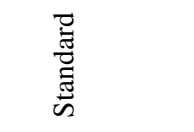 & 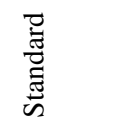 & 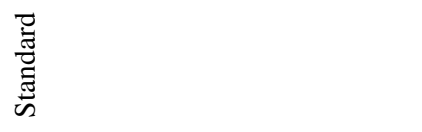 \\
\hline 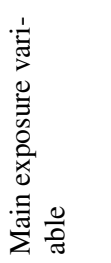 & 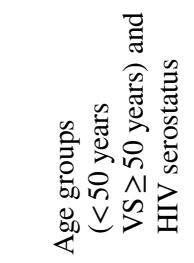 & 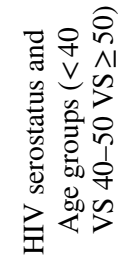 & 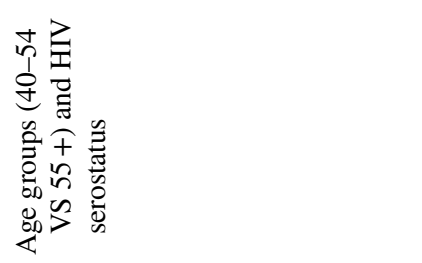 \\
\hline 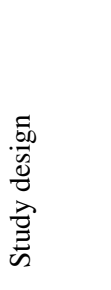 & 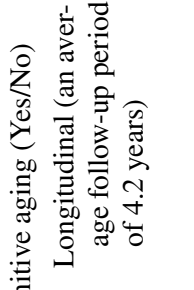 & 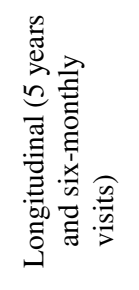 & 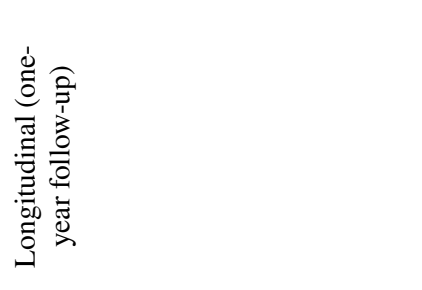 \\
\hline 异 & 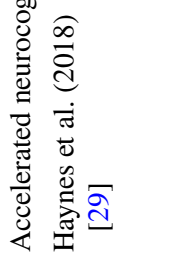 & 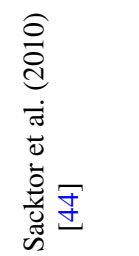 & 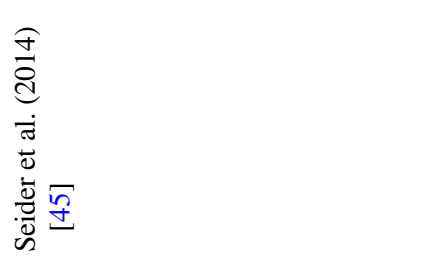 \\
\hline
\end{tabular}




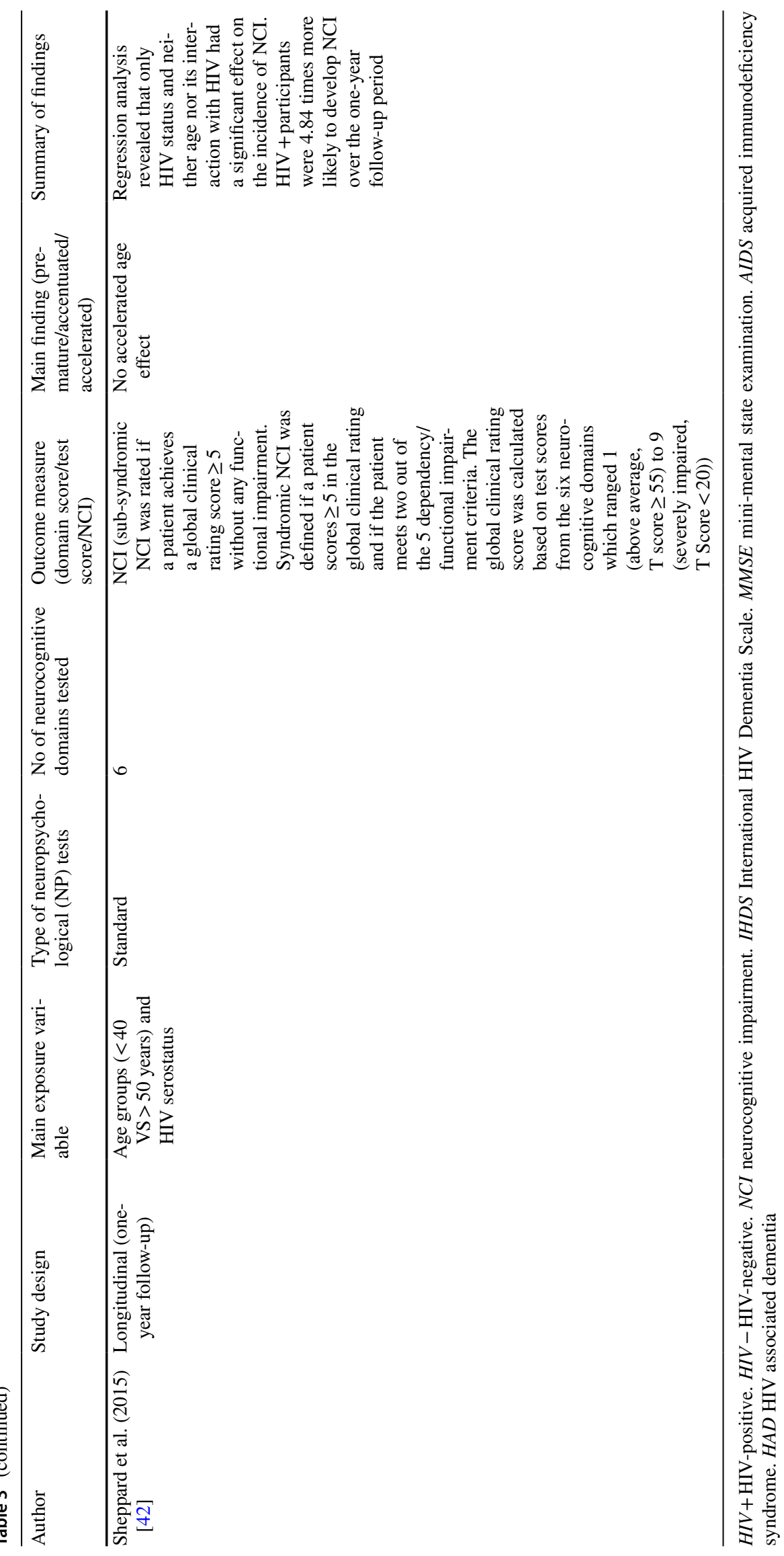




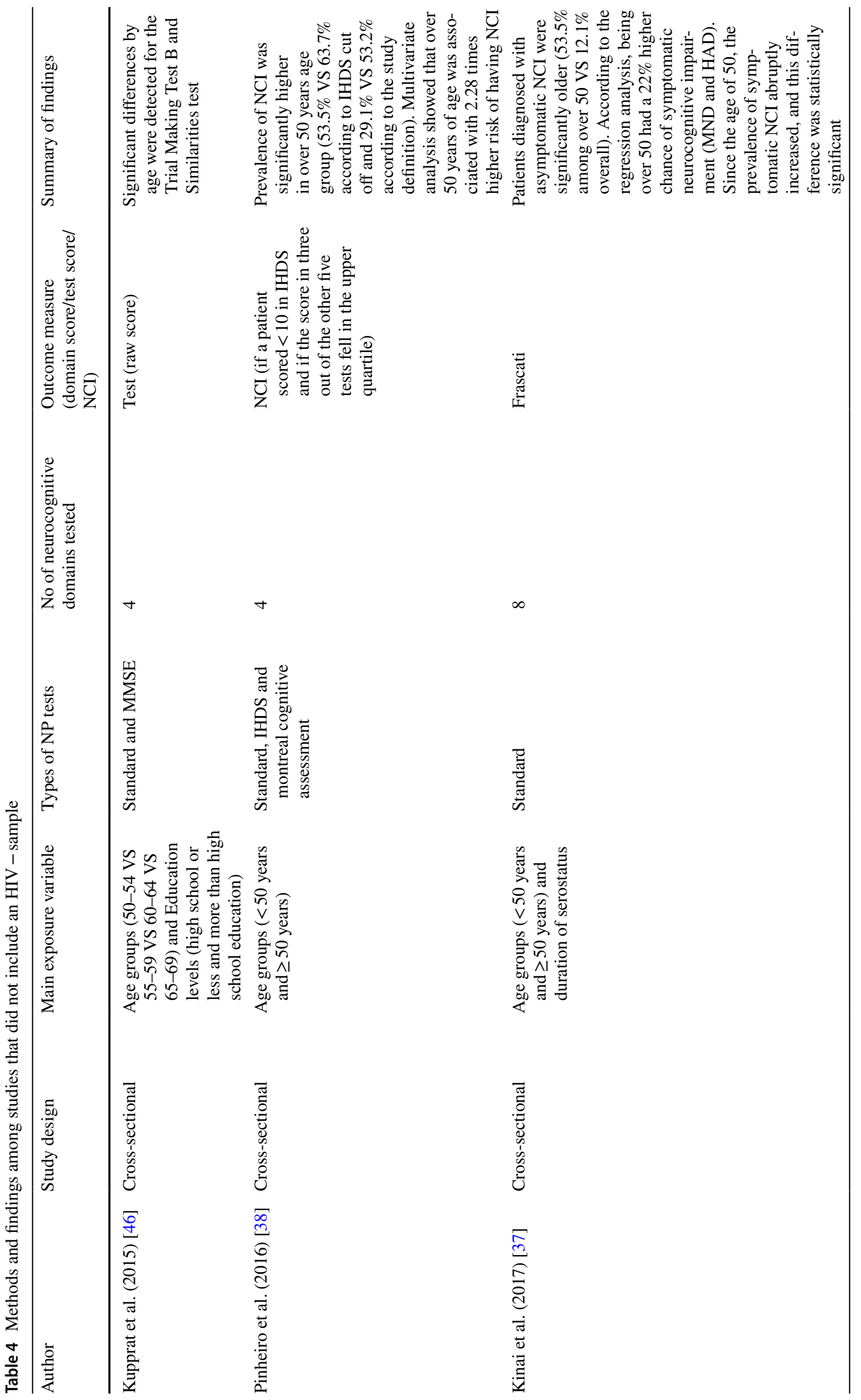




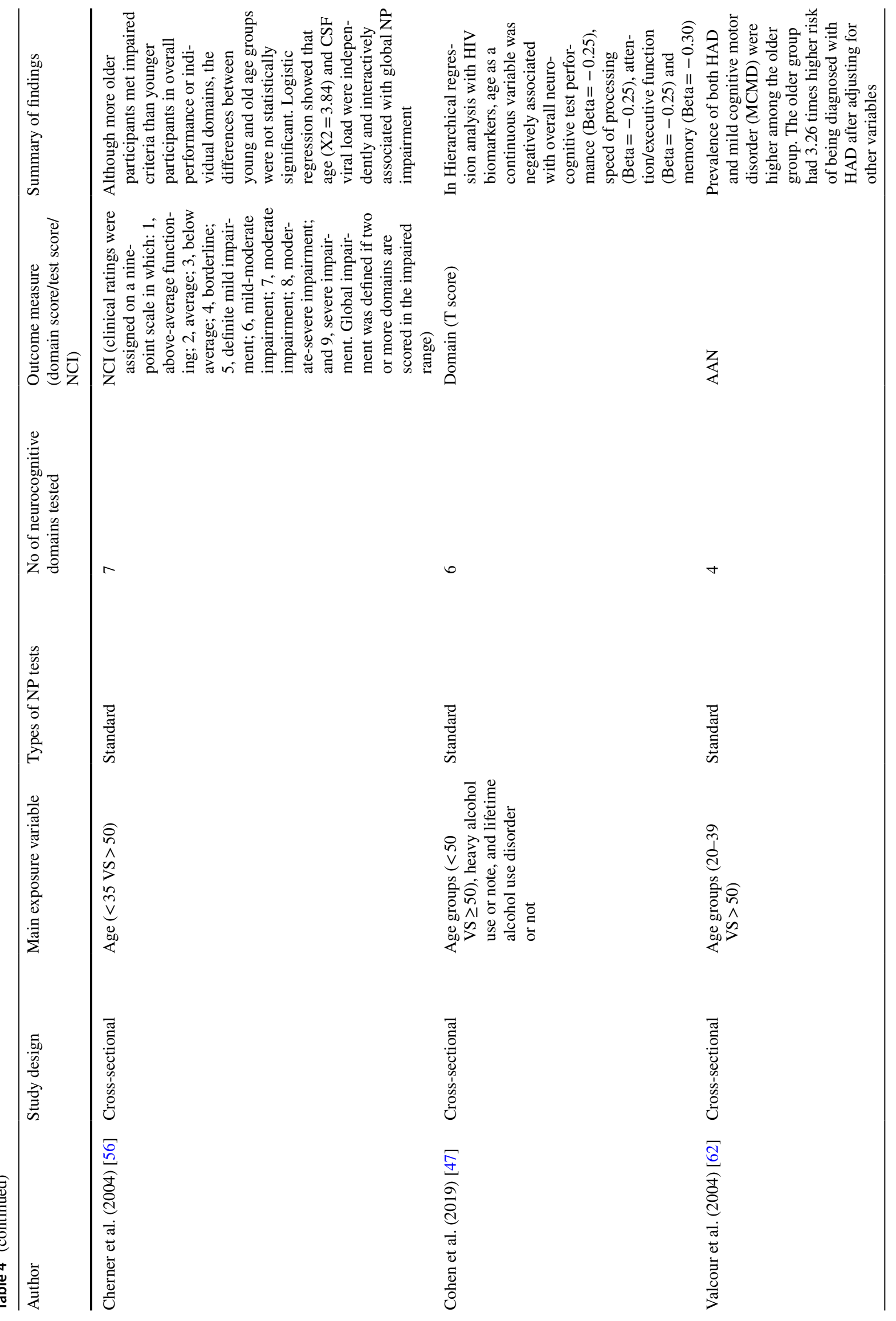




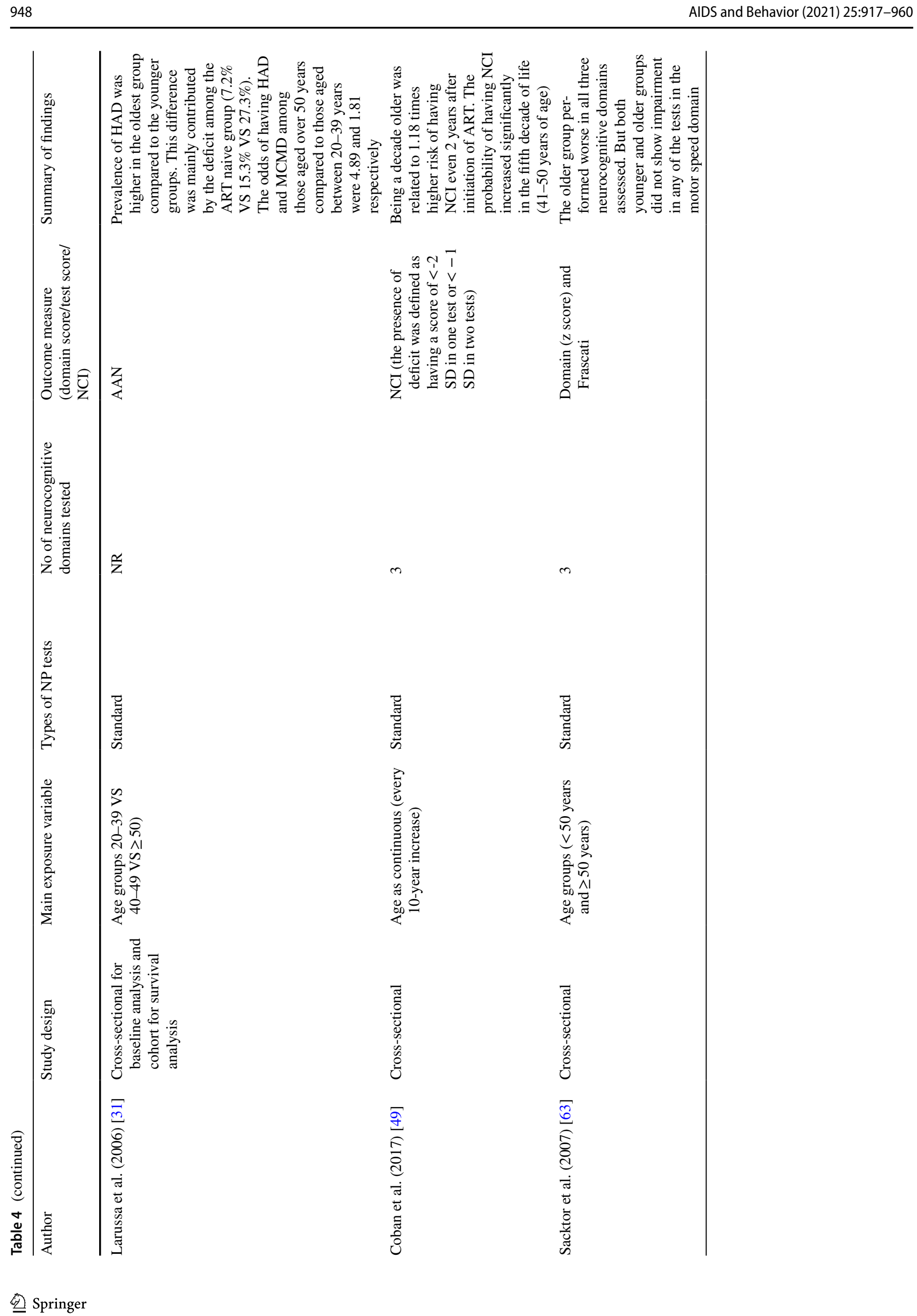




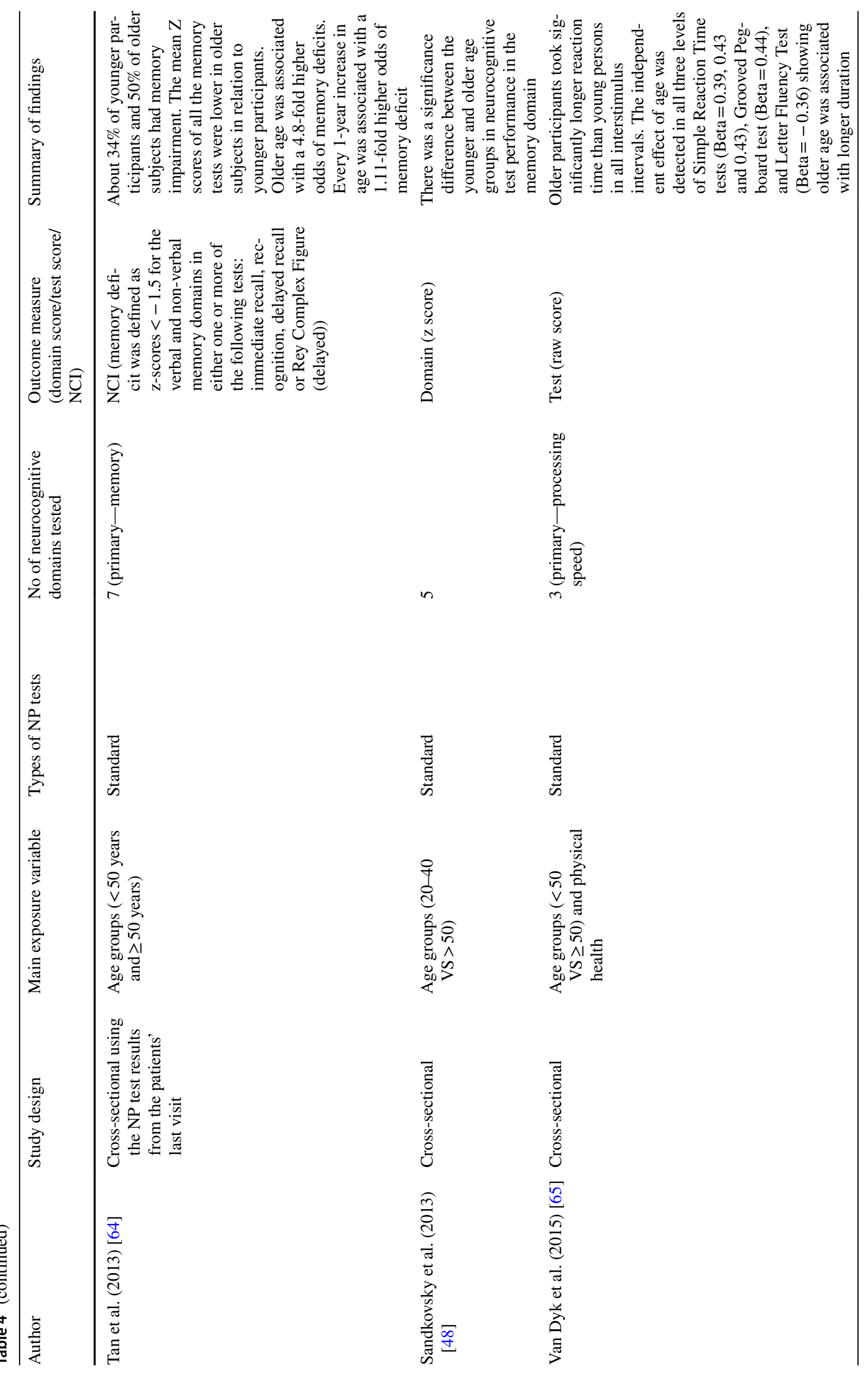




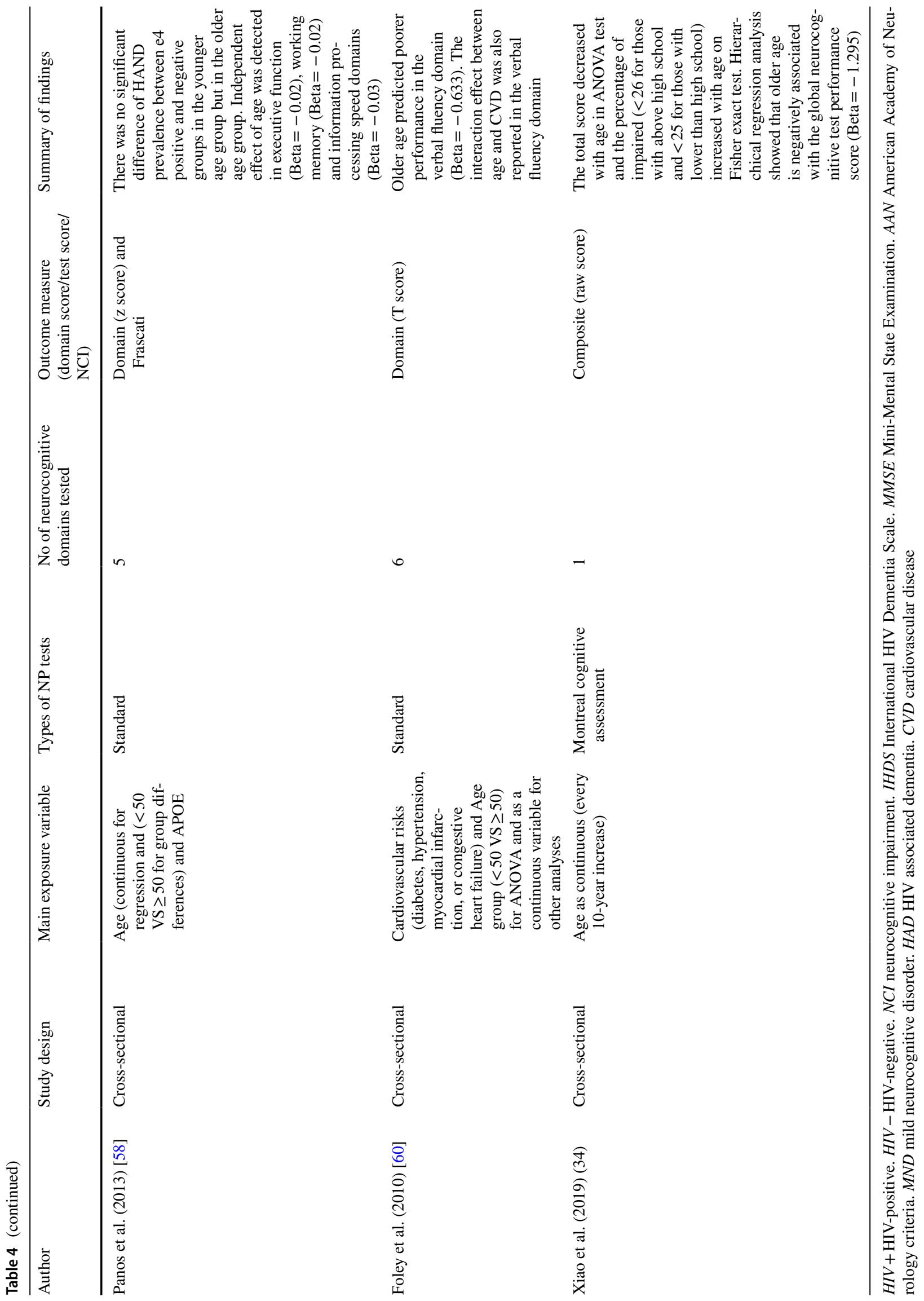


studies, except three studies [32, 40, 45], where the lower bound was 45, 55 and 60 years respectively. Further, the upper bound of the younger age group was not consistent across all the studies. Some studies used $<50$, whereas some studies adopted 35 or 40 years as upper limit of the younger age group. Apart from the three studies that only included participants over 50 years of age, the average proportion of cases over 50 years of age across the rest of the studies was $40 \%$ but with a wide range: $8-61 \%$.

\section{How was the cognition measured and defined?}

All of the studies used comprehensive neuropsychological tests to measure neurocognitive performance, and three studies used screening tests like the International HIV Dementia Scale (IHDS) and Montreal Cognitive Assessment (MoCA) $[33,34,38]$. Only six $[32,35,42,50,54,59]$ out of 22 studies with HIV- controls and nine [31, 37, 48, 49, 56, 61-64] out of 15 studies without a control group standardized cognitive test scores with normative data. Twenty-six out of 37 studies explored the effect of age on a continuous measure of overall or domain specific neurocognitive performance, while 10 studies assessed the effect of age on study-defined NCI criterion. One study examined the age influence on neuropsychological test scores dispersion [59]. Among the studies that had NCI as a major outcome, two studies [31, 62] used the American Academy of Neurology (AAN) criteria [13] and three studies [37, 42, 56] used the Frascati criteria to define NCI. The remaining five studies adopted customized impairment criteria [32, 38, 40, 49, 64]. In one study [38] that used the IHDS and MOCA, the cut-off for NCI was $<10$ for IHDS and ranked in the first quartile for MOCA. Among studies which measured the age effect on continuous neurocognitive performance, 16 studies analyzed on domain scores while 10 studies evaluated individual test scores.

\section{Global cognition versus cognitive domains}

The majority of studies ( $75 \%$ or $28 / 37$ ) focused on global cognition or multiple cognitive domains. Nine studies selectively assessed age effects on specific cognitive domains, for example, verbal fluency [54] and memory [45, 52, 64], or a group of cognitive functions that underlie a neural pathway [53]. Among studies that focused on overall neurocognitive performance, 2 out of 3 assessed five or more cognitive domains. All the domains covered in the studies are aligned within the neurocognitive domains recommended to be evaluated in the Frascati criteria [13]: "Attentioninformation processing, language, abstraction-executive, complex perceptual motor skills, memory, including learning and recall, and simple motor skills or sensory perceptual abilities".

\section{HAND prevalence}

Only eight out of 36 studies (22\%) reported HAND prevalence [35-37, 39, 53, 58, 62, 64]. Of these, one study reported the prevalence only among older HIV + participants [54]. Seven studies used the Frascati criteria while one study used AAN criteria [62]. The prevalence of HAND varied highly among the reported studies ranging from 15 to $89 \%$. The lowest prevalence was observed in a study [35] that included only high functioning HIV + participants. The highest prevalence rate of HAND was found in a study where a large proportion $(81 \%)$ of HIV + participants had substance use disorders [64]. Four out of eight studies [36, 37, 39, 53] classified HAND clinical sub-categories, with ANI being the most prevalent (range: 53\%$74 \%$ ) in three studies, while MND was the predominant classification in one study (55\%) [39]. Four of the studies compared HAND prevalence between age groups [37, 58, $62,64]$. HAND was found to be higher among younger participants in one study possibly because of higher cognitive reserve among older participants [58].

\section{Statistical methods to assess the age effect}

Statistical methods used to identify the effect of age on neurocognition among HIV + participants (versus HIVwhen appropriate) varied across studies. Chi-square test or Fisher's exact test, t-test or Wilcoxon test and Analysis of Variance (ANOVA) test were used to compare group differences in exploratory analyses depending on the type of predictor variables. Two-way ANOVA or Analysis of Covariance (ANCOVA), linear regression, logistic regression, generalized linear model, and linear mixed model were used for the main analysis to identify the interaction effect of HIV and age on neurocognition among studies which included HIV- controls. In studies without HIV- participants, hierarchical regression, linear regression, logistic regression, generalized estimating equation model, and cox proportional hazard model were adopted as a primary analysis method to identify the effect of age on cognition among PLHIV. The type of neuropsychological scores (i.e. raw scores, T-scores, $\mathrm{z}$-scores) used for analysis also differed across studies. Z-score was the most common type of score (14/37 studies) followed by the raw score (13/37 studies), and T-score (10/37 studies). Attempts were made in all the studies except in one [50] to control for possible confounding factors such as non-age demographic factors, HIV biomarkers (e.g. nadir 
CD4), comorbidities, substance use and depression, and factors which are significantly different between HIV + and HIV-participants.

\section{Risk of bias within studies}

Detailed appraisal outcomes for each question in the questionnaires are presented in Tables 5 and 6 (findings for individual studies are presented in the Supplementary File 3). Among cross-sectional studies, 22/31 studies meet the criteria for "Yes" or "Partly" across all the appraisal questions. Eight studies [34, 38, 46, 50, 51, 58, 60, 65] neither included age-matched HIV- controls nor used demographically corrected cognitive test scores. One study [41] did not give clear inclusion criteria. Confounding factors were not identified and controlled for in another study [50]. Among six longitudinal studies, only one study [31] met the criteria for "Yes" or "Partly" in all the questionnaires. One study [42] did not report how attrition was handled. Four other studies [29, 43-45] did not include age-matched HIV- controls or did not use demographically corrected cognitive test scores.

\section{Narrative synthesis}

\section{Studies with an HIV-control group and the evidence of premature, accentuated and accelerated neurocognitive aging}

Out of 22 studies that included an HIV- sample, 17 studies were cross-sectional and five studies [29, 42-45] were longitudinal. Fifteen cross-sectional studies aimed to find the interaction effect of HIV and age on a continuous measure of neurocognitive test performance whereas two studies [32, 40] examined the interaction effect of HIV and age on both $\mathrm{NCI}$ and neurocognitive test performance scores. Among the five longitudinal studies, four studies tested the interaction effect of HIV and age on neurocognitive test performance while one study [42] assessed the interaction effect of HIV and age on incident NCI. In one of the longitudinal studies, the analysis was designed to test the interaction effect of HIV and age only on cross-sectional neurocognitive test performance rather than neurocognitive decline across the study period [43]. Two longitudinal studies [44, 45] examined the interaction effect of HIV and age on both cross-sectional and longitudinal neurocognitive test performance.

Premature neurocognitive aging effect was examined in 17 cross-sectional studies and three longitudinal studies that examined the HIV and age interaction effect on crosssectional neurocognitive test performance. Of these studies, 9/20 (45\%; eight cross-sectional and one longitudinal) showed evidence of premature neurocognitive aging. In all but one study [53], this was attributed to the significantly inferior performance of older HIV + compared to the HIVparticipants. In one study [53], the interaction effect came from the superior performance of younger HIV-participants.

Accentuated neurocognitive aging effect was investigated in two cross-sectional studies, which tested the HIV and age interaction effect on the number of tests performed under a defined cut-off score. Neither of these studies found evidence of accentuated neurocognitive aging.

Accelerated neurocognitive aging effect was assessed in four longitudinal studies that analyzed the HIV and age interaction effect on longitudinal neurocognitive test performance as decline in performance across the study period. Accelerated aging was detected in three studies (75\%), where neurocognitive decline was significantly greater among older HIV + compared to HIV- individuals [29, 44, 45].

Among 10 studies that did not observe any of the abnormal patterns of neurocognitive aging, the poorest neurocognitive test performance was still observed among older HIV + participants in two of the studies [32, 66].

In evaluating whether study and sample characteristics were related to any of the abnormal neurocognitive aging effects, we found that longitudinally designed studies were more likely to observe premature or accelerated neurocognitive aging effect ( $80 \%$ versus $47 \%$ ). Sample size also appeared to contribute to the outcomes of interest. Indeed, the three studies [33, 43, 44] that had sample sizes of $\mathrm{N}>500$, all found evidence for premature or accelerated neurocognitive aging. In studies testing premature aging $(\mathrm{N}=20)$, the median sample size was 189 (IQR: 132.25-304.25); in studies testing accentuated aging $(\mathrm{N}=2)$, the median sample size was 216.5 (IQR:192-241); and in the longitudinal studies assessing accelerated aging $(\mathrm{N}=4)$, the median sample size was 115 (IQR: 62.25-993.5). These figures highlighted that sample sizes in majority of studies are below the required sample size of 350 for premature aging, 1,050 for accentuated aging, and 230 for accelerated aging (see Fig. 2 for detailed sample size computations with G*Power 3.1 [67]) to achieve a conventional power of $80 \%$ in order to detect a small-medium effect size of the chronological age effect. Percentage of older people in the total participants also seemed to be associated with whether the hypothesized neurocognitive aging effect was observed or not. Indeed, when $\geq 50 \%$ of the sample were over 50 years of age, $67 \%$ of studies reported the hypothesized neurocognitive aging effect compared to only $50 \%$ of studies where less than $50 \%$ of samples were over 50 years. Studies concentrating on NCI rather than on overall neurocognitive test performance were less likely to find the neurocognitive aging effect (i.e., accentuated or accelerated aging). As such, none out of three studies [32, 40, 42] which focused on NCI found abnormal neurocognitive aging.

HIV disease characteristics may also be related to whether premature, accentuated, and/or accelerated neurocognitive 
aging effects were observed. Indeed, 3/4 studies that recruited only HIV + participants with viral suppression did not find any abnormal neurocognitive aging effect. In studies where standard HIV disease staging was used (WHO or $\mathrm{CDC}$ ), a higher proportion that reported abnormal neurocognitive aging effects than those that did not $(71 \%$ versus $33 \%$ ) included HIV + participants, with at least $50 \%$ being diagnosed with a WHO Stage 4 or CDC Stage C. In terms of nadir CD4 + T cell count, a higher percentage of studies that found abnormal neurocognitive aging effects compared to those that did not (56\% versus $38 \%$ ) was composed of HIV + individuals with an average nadir CD4 + T cell count $<200$ copies $/ \mathrm{ml}$. A greater proportion of studies that showed abnormal patterns of neurocognitive aging $(73 \%$ vs $50 \%$ ) also reported a mean/median duration of HIV infection $\geq 12$ years among their HIV + participants. Finally, among the seven studies which reported that depressive symptoms were significantly more common among PLHIV than HIV- controls, six studies (86\%) observed that abnormal patterns of neurocognitive aging were present.

Table 5 Rating on quality appraisal among cross-sectional studies with the JBI tools

\begin{tabular}{|c|c|c|c|c|}
\hline & Yes $(\%)$ & Partly (\%) & No $(\%)$ & NA $(\%)$ \\
\hline Were the criteria for inclusion in the sample clearly defined? & 97 & 0 & 3 & 0 \\
\hline Were the study subjects and the setting described in detail? & 90 & 10 & 0 & 0 \\
\hline Was the exposure measured in a valid and reliable way? & 97 & 3 & 0 & 0 \\
\hline Were objective, standard criteria used for measurement of the condition? & 39 & 29 & 0 & 35 \\
\hline Were confounding factors identified? & 81 & 16 & 3 & 0 \\
\hline Were strategies to deal with confounding factors stated? & 81 & 16 & 3 & 0 \\
\hline Were the outcomes measured in a valid and reliable way? & 87 & 10 & 0 & 3 \\
\hline Was appropriate statistical analysis used? & 87 & 13 & 0 & 0 \\
\hline $\begin{array}{l}\text { Were demographically comparable HIV negative controls included and/or were } \\
\text { demographically corrected cognitive scores used? }\end{array}$ & 61 & 13 & 26 & 0 \\
\hline
\end{tabular}

"Yes" means the study fully meets the criteria for this question. "Partly" means the study only partly meets the criteria. "No" means the study does not meet the criteria for this question at all. "NA" means this question is not applicable to this study

In the review context, we defined confounding factors as major neurological or psychiatric conditions. Age-related conditions were not considered under this item as all studies did not assess this question optimally, so this represents an overall limitation for the current HIV and cognitive aging literature at this moment in time

Table 6 Rating on quality appraisal among longitudinal studies with the JBI tools

\begin{tabular}{|c|c|c|c|c|}
\hline & Yes $(\%)$ & Partly $(\%)$ & No $(\%)$ & NA (\%) \\
\hline Were the two groups similar and recruited from the same population? & 83 & 0 & 0 & 17 \\
\hline Were the exposures measured similarly to assign people to both exposed and unexposed groups? & 83 & 0 & 0 & 17 \\
\hline Was the exposure measured in a valid and reliable way? & 100 & 0 & 0 & 0 \\
\hline Were objective, standard criteria used for measurement of the condition? & 17 & 17 & 0 & 67 \\
\hline Were confounding factors identified? & 83 & 17 & 0 & 0 \\
\hline Were strategies to deal with confounding factors stated? & 83 & 17 & 0 & 0 \\
\hline $\begin{array}{l}\text { Were the groups/participants free of the outcome at the start of the study (or at the moment of expo- } \\
\text { sure)? }\end{array}$ & 17 & 0 & 0 & 83 \\
\hline Were the outcomes measured in a valid and reliable way? & 100 & 0 & 0 & 0 \\
\hline Was the follow up time reported and sufficient to be long enough for outcomes to occur? & 83 & 0 & 0 & 17 \\
\hline Was follow up complete, and if not, were the reasons to loss to follow up described and explored? & 50 & 17 & 0 & 33 \\
\hline Were strategies to address incomplete follow up utilized? & 33 & 0 & 17 & 50 \\
\hline Was appropriate statistical analysis used? & 100 & 0 & 0 & 0 \\
\hline $\begin{array}{l}\text { Were demographically comparable HIV negative controls included and/or were demographically cor- } \\
\text { rected cognitive scores used? }\end{array}$ & 33 & 0 & 67 & 0 \\
\hline
\end{tabular}

"Yes" means the study fully meets the criteria for this question. "Partly" means the study only partly meets the criteria. "No" means the study does not meet the criteria for this question at all. "NA" means this question is not applicable to this study 
A premature aging

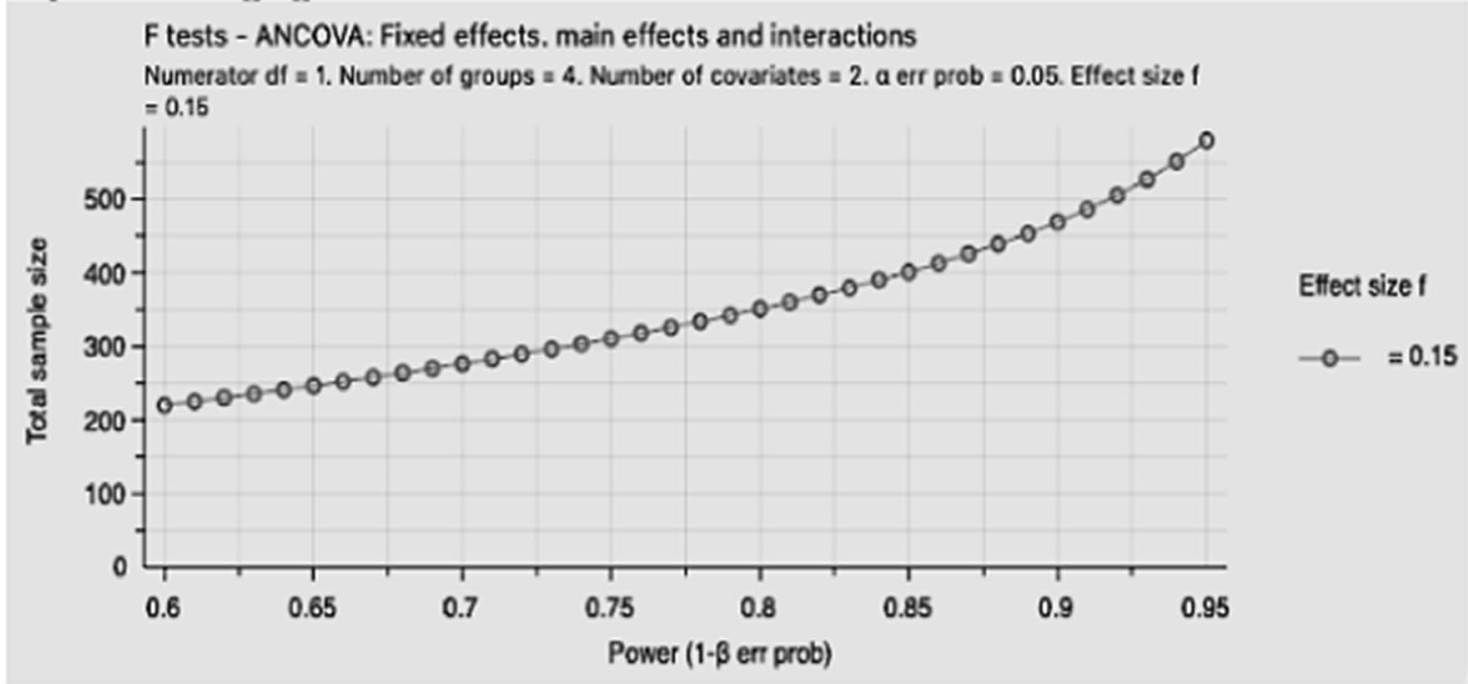

B accentuated aging

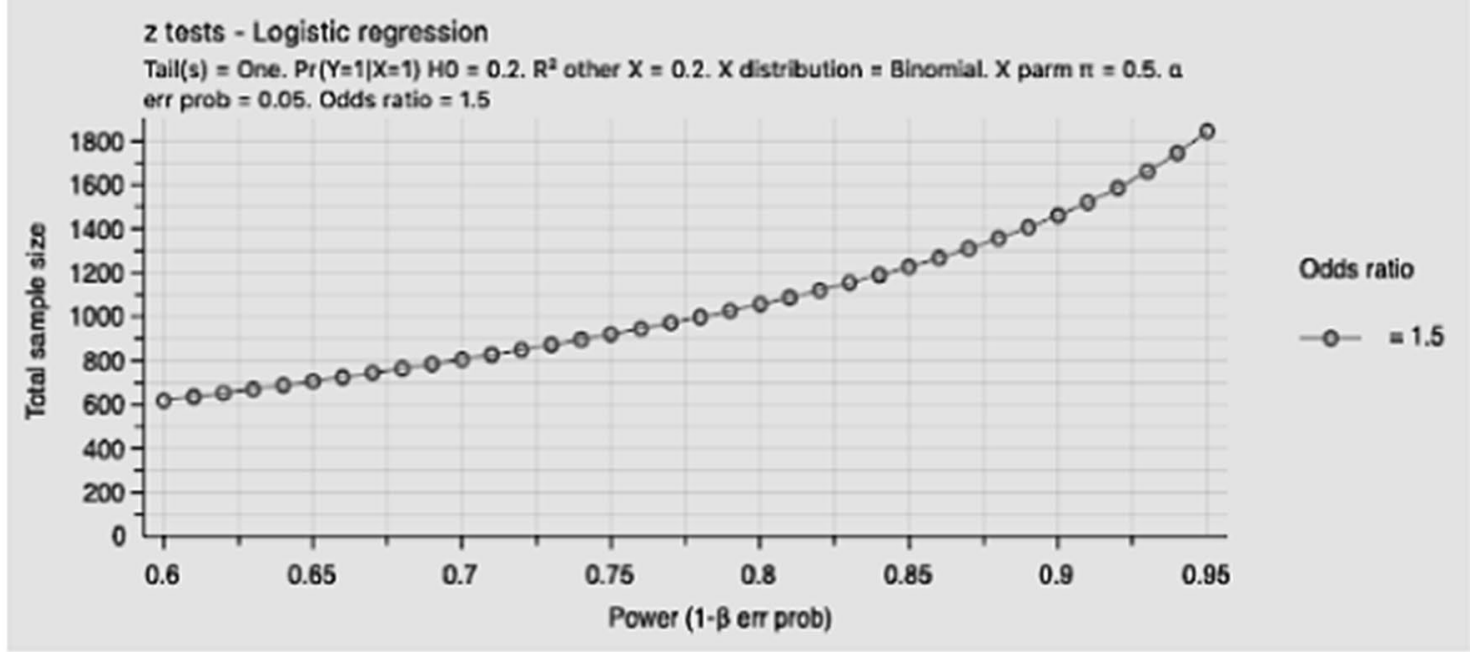

\section{$\mathrm{C}$ accelerated aging}

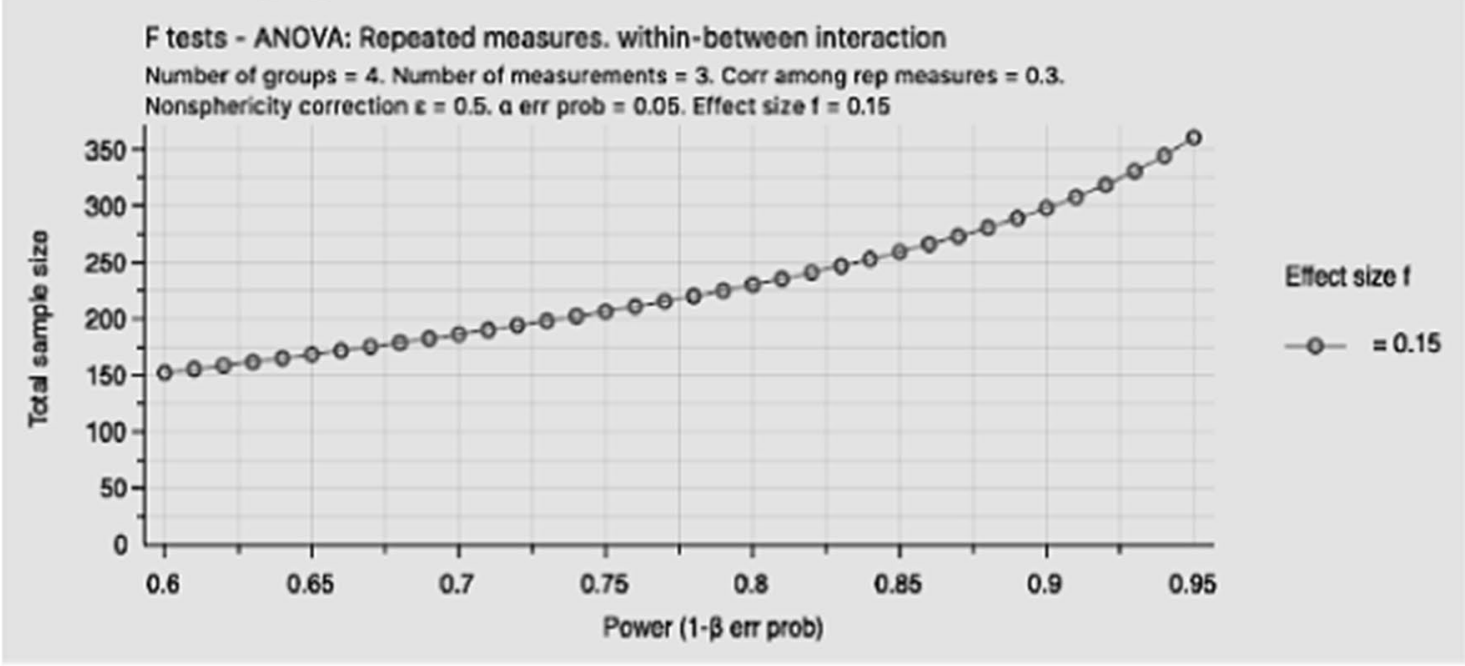


४Fig. 2 Sample size estimation to detect a small-medium effect size of premature and accentuated aging in a sample statistical model. $\mathrm{G}^{*}$ Power 3.1 was used for these calculations. For premature aging, we assume a small-medium effect size, $F=0.15$. Four groups are younger HIV-, older HIV-, younger HIV +, and older HIV +. The number of covariates is referred from the median number of covariates among studies that have assessed premature neurocognitive aging. For accentuated aging we assume odds ratio $=1.5$ (independent age effect) and $20 \%$ of explanatory variance from other covariates. For accelerated aging, we assume the same effect size and the same number of groups as in premature aging with 3 testing, a correlation among repeated measures $(\mathrm{r}=.3)$, and a nonsphericity correction at 0.5 representing variations in test retest variance

\section{Studies without an HIV-controls group and the magnitude of the age effect on cognition}

Among 15 studies that did not include HIV-participants, the presence of an effect of age on neurocognition was observed among the HIV + participants in all the studies. In studies with $\mathrm{NCI}(\mathrm{N}=7)$ as the outcome, the odds ratio (OR) of having NCI among older HIV + compared to their younger counterparts varied between 1.18 and 4.8 indicating a higher risk of NCI among older PLHIV compared to the younger people. The effect sizes among the studies which used continuous neurocognitive performance are not reported because most of these studies did not use demographicallycorrected cognitive scores.

\section{Discussion}

This is the first systematic review in the area of HIV and neurocognitive aging. This review exhaustively searched four scientific databases to find the relevant literature and determined the current known evidence of premature, accentuated, and accelerated neurocognitive aging among PLHIV and its associated factors by critically and independently appraising the 37 eligible articles. Our review also provides strong evidence of major limitations in the selected studies.

We found that $62 \%$ studies met the criteria fully or partly for all the quality assessment items evaluated with the JBI tools. Previous non-systematic reviews have not evaluated study quality with standard quality tools. However, the studies' heterogeneity in design (cross-sectional/longitudinal), operationalization of age as a predictor, definition of $\mathrm{NCI}$ and continuous neuropsychological outcomes (global/ domain/focused, various types of tests and various test-score standardization methods), in addition to the statistical and conceptual operationalization of aging effects on neurocognition did not permit for a meta-analysis. This represents the number one limitation across this research literature. One of the most illustrative aspects in the lack of harmonization across this literature is that despite the availability of standard diagnostic criteria for HAND [13, 68], only $22 \%$ of studies used these criteria to report HAND prevalence. This is true, despite the majority of studies (68\%) using large enough test batteries to compute the neurocognitive severity, part of the criteria.

The second major limitation is that $14 / 37$ studies (38\%) included no control samples. It should be clearly stated that while the age effect can be tested on neurocognition in studies without a control group, these studies are fundamentally hindered in their capacity to test premature, accentuated and accelerated aging effects. In other words, these studies offer limited information as to whether PLHIV are at a greater risk of abnormal brain aging and ultimately dementia. In addition, quality appraisal with the JBI tools found that in six of the studies with a control group age was not comparable between HIV + and HIV- groups and demographically-corrected cognitive scores were not used. Six of the studies that did not include a control group also did not use demographically-corrected cognitive scores. To differentiate cognitive aging effects due to HIV from normal aging effect, HIV- controls need to be matched with HIV + participants, at least in age and preferably in other demographic factors as well. If HIV- controls are not age-comparable or if a control group is not included and the question is about whether there is an abnormally large aging effect in PLHIV, then demographically corrected cognitive scores need to be used.

The neurocognitive aging effects in studies that did not include an HIV- control group were consistent but had a wide range. The wide range of effect suggests that the prevalence of HAND and associated HIV disease factors are widely heterogeneous across studies. The higher risk of NCI among older PLHIV compared to younger PLHIV may be attributed to greater exposure to other risk factors of HAND such as a diagnosis with a more severe HIV clinical disease stage, living with HIV without ART for a longer duration, low baseline CD4 count, and exposure to neurotoxic ART drugs [24]. On the other hand, there are likely some embedded survivor effects that have not been taken into account [12].

Previous non-systematic reviews of the HIV and neurocognitive aging literature [22-24] showed that premature and accelerated neurocognitive aging effects were inconsistent, while the accentuated aging was not conceptualized as a separate effect of interest. Using systematic definitions for each abnormal patterns of neurocognitive aging, our systematic review provides a systematic appraisal of this inconsistency. Among the studies that have investigated premature aging $(\mathrm{N}=20), 45 \%$ reported premature aging. No study found evidence of accentuated aging, though this was only investigated in two studies representing another major limitation of the current HIV neurocognitive aging research. Among the studies that investigated accelerated neurocognitive aging $(\mathrm{N}=4), 75 \%$ reported accelerated aging. Our systematic review also found that study and 
sample characteristics were associated with the likelihood of reporting abnormal neurocognitive aging. In the following section, we discuss each of these findings in detail.

This review showed that all three studies (two longitudinal and one cross-sectional) where sample size was greater than 500 reported an abnormal pattern of cognitive aging effect. The lack of large enough sample sizes to detect smallmedium effect sizes in most other studies as assessed by our sample size computation could account for inconsistencies in the detection of abnormal patterns of cognitive aging. This represents a major issue especially when we consider that cognitive aging in PLHIV is multifactorial [69]. Besides the effect of chronological age itself, other factors such as HIV characteristics, resilience, lifestyle factors, mental health, age-related comorbidity burden, and aged-related genetic factors need to be individually considered, in addition to their interactions (including complex interaction levels, such as full factorial model and/or polynomials). The current studies typically included 1-3 covariates and thus did not address multi-factorial aging effects. A dataset which would take into account multi-dimensional aging effects would lead to a high-dimensional dataset that typically requires very large sample size whether it is for traditional statistical analyses or data-driven methods (e.g., machine learning) [70]. Reaching such a sample size may only be feasible by prospectively co-enrolling HIV + and HIV- participants in the multitude of existing NeuroHIV studies worldwide. This is not an unrealistic effort, considering it is in progress in dementia research such as International Centenarian Consortium-dementia [71] or neuroimaging research (e.g. Alzheimer's Disease Neuroimaging Initiative) [72] to cite a few examples. However, data and methods harmonization are a pre-requisite [73].

As already recommended in the standard diagnostic criteria for HAND [13], core minimal data requirements for data pooling should include the coverage of at least five neurocognitive domains, and collection of major confounding conditions such as major neurological and psychiatric disorders, mood disorders, substance/alcohol use disorder. This is not trivial as some of the reviewed studies did not report on HIV characteristics and other major confounders. Standardization in the collection, operationalization and scoring of these data would also be needed, which represents a major effort.

What would constitute an even greater effort in the data pooling is the addition of age-related conditions. In the current review, only a few studies collected information on age-related comorbidities such as CVD (4/37 studies) and hypertension (10/37 studies), which represents one of the most important limitations of the current literature in terms of its immediate clinical relevance. As PLHIV age, we know that comorbidities will play a greater role on neurocognitive decline in addition to chronological age itself [74]. In dementia research, there is increasing recognition that preventable mid-life conditions all represent a cumulative risk factor for dementia [75]. From a clinical perspective, it is imperative to understand the contributions of these comorbidities on NCI among PLHIV for the development of better prevention and clinical management strategies [76], as it may represent one of the best ways to reduce dementia risk in PLHIV. This may also help understanding what the dementia risk may be in PLHIV with low comorbidity burden.

The availability of this kind of data pooling and sharing system could be globally promoted as this is in line with NIH policies and other public research funding agencies around the world [77]. This mechanism would be especially beneficial to smaller studies from resource-limited settings and low-medium income countries with emerging aging HIV populations [78]. In this review, a major limitation is that no study was from low income countries and no research has been conducted on HIV and neurocognitive aging in sub-Saharan Africa [79], where HIV rates are the highest globally [80], where there is an aging HIV population with historical AIDS and rising multi-comorbidities $[81,82]$, and where HAND prevalence rates range from $16 \%-80 \%$ [83].

In order for this sharing platform to be effective internationally, the NeuroHIV community should agree on a core neuropsychological test battery allowing for the use of HAND diagnostic criteria that can be applied across countries with relative ease. A major challenge with the current diagnostic criteria is the requirement of a functional assessment, and that there is currently not a culturally appropriate functional scale for use in many low-medium income settings. Therefore, an improved algorithm compared to what has been published in 2007 [13] that includes a crossculturally valid functional assessment would enable more comparable research in the future [14]. Standardization in the collection, operationalization and scoring of these data would also be needed.

The proportion of older persons (over 50 years old) in the total sample may have had some effect on whether abnormal patterns of neurocognitive aging were detected. A higher proportion of studies found the interaction effect of age and HIV on neurocognition when more than $50 \%$ of their participants were older PLHIV. The low proportion of older participants may mean low percentage of participants over 60 years old, the age when normal neurocognitive decline starts to increase in the normal population [84]. The lower number of participants over 60 was reported as a limitation in some of the studies in this review that did not find an interaction effect between HIV and age on neurocognition $[32,36,39,40]$. This limitation across the HIV neurocognitive aging literature further demonstrates that follow-up 
of the established NeuroHIV cohort studies need long-term support.

The clinical characteristics of HIV + participants had some impact on the finding of premature, accentuated, and accelerated neurocognitive aging effects. Abnormal patterns of neurocognitive aging were observed more commonly in studies where HIV + participants had a higher rate of known risk factors for HAND such as CDC Stage C or WHO Clinical Stage IV and lower baseline CD4 count $(<200)$. This review also identified the possible "age-duration effect" [19]. Studies which included HIV + participants with longer duration of HIV infection tended to find an interaction effect between HIV and age. This indicates that even between two groups of older HIV + persons with similar age, the group with longer duration of HIV may have lower neurocognitive test performance. Indeed, HIV + persons who have lived with HIV for a longer period of time may have had to experience longer duration of chronic immune activation and inflammation [85]. Future HIV and neurocognitive aging studies should incorporate the duration of HIV infection as a covariate in their analysis in order to be able to differentiate whether abnormal patterns of neurocognitive aging are dependent on either or both chronological age and/or duration of HIV infection.

Studies where HIV + participants had a higher prevalence of depressive symptoms than HIV- participants tended to find abnormal patterns of neurocognitive aging. This is in line with findings that showed that mood disorders may accelerate brain aging [86, 87], and are known independent risk factors for dementia [87]. Because most studies excluded PLHIV with more severe form of psychiatric distress, the neurocognitive aging profile in PLHIV in those with high psychiatric burden remains unknown, and this represents another shortcoming from the current literature. From a clinical perspective, continued investment in bettering the mental health of PLHIV should become a central part of their care as they age.

Our systematic review has the following limitations. We recognize that this review included only articles from peerreviewed journals which were written in English, and that this may have led to a selection bias. However, NeuroHIV literature has been traditionally published in English, even when from non-English speaking countries, therefore the bias is limited. Furthermore, it is possible that more negative findings exist but were not published. This effect is potentially moderated by the fact that the question of premature, accentuated and accelerated neurocognitive aging among PLHIV is highly debated $[22,88]$, and thus, that negative findings have been considered as important as positive findings. Our review concentrated on neurocognitive rather than brain aging (e.g., imaging studies) and it is possible that brain changes in some instances precede neurocognitive aging. As some of the studies in this review included only
MSM participants (6 studies) and male participants (2 studies), the findings may not fully be relevant to female HIV populations. Since none of studies came from low-income countries, our findings may not be directly applicable to PLHIV from low-income countries. We conceptualized aging as chronological age, however as we have discussed above, aging is a multifactorial process that should ideally be captured multi-dimensionally. Our review shows however, that the current state of the HIV and neurocognitive aging literature would have not permitted the inclusion of other makers of aging.

To conclude, the proportion of PLHIV reaching 60 years of age is ever increasing, an age at which dementia prevalence starts to rise in the general population. Importantly age is the \#1 risk factor for dementia. Therefore, even small effects of premature, accentuated or accelerated neurocognitive aging in PLHIV may have major public health implications for dementia risk at the global HIV population level. Evidence for premature neurocognitive aging was inconsistent. Evidence for accelerated neurocognitive aging was consistent but was based on a small number of studies, albeit the largest. Evidence for accentuated neurocognitive aging was rarely investigated and negative when tested. A stronger level of evidence is critically needed to inform clinicians and the HIV care sector about dementia risk among PLHIV to properly facilitate dementia screening, prevention and treatment among aging PLHIV. We propose that to determine accurate estimates of abnormal patterns of neurocognitive aging in PLHIV at the meta-analytical and epidemiological levels, an international effort should be made to collect harmonized longitudinal neurocognitive and other relevant data (including selected key neurological, psychiatric, agecomorbidities and other age markers, that is a high-dimensional dataset) in a very large number. This is needed to enable testing typical small-to medium chronological age effect $(d=0.3)$ on standard neuropsychological testing [89]. Ideally, this sample would be composed of PLHIV and local age-matched controls that comprise a good representation of low- and middle-income countries and at least $50 \%$ with age over $50+$ years old.

Author contributions HLA, BJB, LAC: Conceptualization; HLA, LAC: Methodology; HLA, MA, LAC: Formal Analysis and investigation; HLA: Writing — original draft preparation; HLA, MA, HG, RNR, BB, LM, LAC: Writing-review and editing; RNR, BB, LM, LAC: Supervision.

Funding Dr Aung receives a primary scholarship from the University of New South Wales and a top up scholarship from NeuRA to study his $\mathrm{PhD}$. This systematic review is part of his $\mathrm{PhD}$ project: Global Health Impact of Brain Aging among HIV Infected Persons. 


\section{Compliance with ethical standards}

Conflict of interest All authors declare that they have no conflict of interest.

Open Access This article is licensed under a Creative Commons Attribution 4.0 International License, which permits use, sharing, adaptation, distribution and reproduction in any medium or format, as long as you give appropriate credit to the original author(s) and the source, provide a link to the Creative Commons licence, and indicate if changes were made. The images or other third party material in this article are included in the article's Creative Commons licence, unless indicated otherwise in a credit line to the material. If material is not included in the article's Creative Commons licence and your intended use is not permitted by statutory regulation or exceeds the permitted use, you will need to obtain permission directly from the copyright holder. To view a copy of this licence, visit http://creativecommons.org/licenses/by/4.0/.

\section{References}

1. Teeraananchai S, Kerr S, Amin J, Ruxrungtham K, Law M. Life expectancy of HIV-positive people after starting combination antiretroviral therapy: a meta-analysis. HIV Med. 2017;18(4):256-66.

2. Lima VD, Harrigan R, Bangsberg DR, Hogg RS, Gross R, Yip $\mathrm{B}$, et al. The combined effect of modern highly active antiretroviral therapy regimens and adherence on mortality over time. $\mathrm{J}$ Acquired Immune Defic Syndr. 2009;50(5):529.

3. Costagliola D. Demographics of HIV and aging. Curr Opin HIV AIDS. 2014;9(4):294-301

4. Battegay M, Elzi L. Morbidity and mortality in HIV-infected individuals-A shift towards comorbidities. Swiss Med Wkly. 2009;139(39):564.

5. UNAIDS. HIV and ageing. Geneva: UNAIDS; 2017.

6. Negredo E, Back D, Blanco JR, Blanco J, Erlandson KM, Garolera M, et al. Aging in HIV-infected subjects: a new scenario and a new view. Biomed Res Int. 2017;2017:5897298.

7. UNAIDS. People aged 50+ living with HIV. UNAIDS; 2019

8. Aung HL, Kootar S, Gates TM, Brew BJ, Cysique LA. How alltype dementia risk factors and modifiable risk interventions may be relevant to the first-generation aging with HIV infection? Eur Geriatr Med. 2019. https://doi.org/10.1007/s41999-019-00164 $-6$.

9. Van Epps P, Kalayjian RC. Human immunodeficiency virus and aging in the era of effective antiretroviral therapy. Infect Dis Clin North Am. 2017;31(4):791-810.

10. Brooks JT, Buchacz K, Gebo KA, Mermin J. HIV infection and older Americans: the public health perspective. Am J Public Health. 2012;102(8):1516-26.

11. Wing EJ. HIV and aging. Int J Infect Dis. 2016;53:61-8.

12. Justice AC. HIV and aging: time for a new paradigm. Curr HIV/ AIDS Rep. 2010;7(2):69-766.

13. Antinori A, Arendt G, Becker JT, Brew BJ, Byrd DA, Cherner $\mathrm{M}$, et al. Updated research nosology for HIV-associated neurocognitive disorders. Neurology. 2007;69(18):1789-99.

14. Saloner R, Cysique LA. HIV-associated neurocognitive disorders: a global perspective. J Int Neuropsychol Soc. 2017;23(9-10):860-9.

15. Eggers C, Arendt G, Hahn K, Husstedt IW, Maschke M, NeuenJacob E, et al. HIV-1-associated neurocognitive disorder: epidemiology, pathogenesis, diagnosis, and treatment. J Neurol. 2017;264(8):1715-27.
16. Simioni S, Cavassini M, Annoni JM, Rimbault Abraham A, Bourquin I, Schiffer V, et al. Cognitive dysfunction in HIV patients despite long-standing suppression of viremia. AIDS. 2010;24(9):1243-50.

17. Heaton RK, Franklin DR, Ellis RJ, McCutchan JA, Letendre SL, Leblanc S, et al. HIV-associated neurocognitive disorders before and during the era of combination antiretroviral therapy: differences in rates, nature, and predictors. J Neurovirol. 2011;17(1):3-16

18. Sacktor N, Skolasky RL, Seaberg E, Munro C, Becker JT, Martin E, et al. Prevalence of HIV-associated neurocognitive disorders in the multicenter AIDS Cohort Study. Neurology. 2016;86(4):334-40.

19. Smail RC, Brew BJ. Chapter 7-HIV-associated neurocognitive disorder. In: Brew BJ, editor. Handb Clin Neurol. 2018;152:75-97

20. Harezlak J, Buchthal S, Taylor M, Schifitto G, Zhong J, Daar E, et al. Persistence of hiv- associated cognitive impairment, inflammation and neuronal injury in era of highly active antiretroviral treatment. AIDS (London, England). 2011;25(5):625.

21. Aberg JA. Aging, inflammation, and HIV infection. Top Antivir Med. 2012;20(3):101.

22. Cysique LA, Brew BJ. The effects of HIV and aging on brain functions: proposing a research framework and update on last 3 years' findings. Curr Opin HIV AIDS. 2014;9(4):355-64.

23. Hardy DJ, Vance DE. The neuropsychology of HIV/AIDS in older adults. Neuropsychol Rev. 2009;19(2):263.

24. Wendelken LA, Valcour V. Impact of HIV and aging on neuropsychological function. J Neurovirol. 2012;18(4):256-63.

25. Gates TM, Cysique LA. The chronicity of HIV infection should drive the research strategy of NeuroHIV treatment studies: a critical review. CNS Drugs. 2016;30(1):53-69.

26. Moher D, Liberati A, Tetzlaff J, Altman DG. Preferred reporting items for systematic reviews and meta-analyses: the PRISMA statement. Ann Intern Med. 2009;151(4):264-9.

27. Institute JB. The Joanna Briggs Institute Critical Appraisal tools for use in JBI Systematic Reviews Adelaide, SA2017 [Available from: https://joannabriggs.org/research/critical-appraisal-tools .html.

28. Popay J, Roberts H, Sowden A, Petticrew M, Arai L, Rodgers $\mathrm{M}$, et al. Guidance on the conduct of narrative synthesis in systematic reviews: a product from the ESRC Methods Programme. Lancaster: Lancaster University. 2006;10(2.1):1018.4643.

29. Haynes BI, Pitkanen M, Kulasegaram R, Casey SJ, Schutte M, Towgood K, et al. HIV: ageing, cognition and neuroimaging at 4-year follow-up. HIV Med. 2018;19(6):376-85.

30. Towgood KJ, Pitkanen M, Kulasegaram R, Fradera A, Kumar A, Soni S, et al. Mapping the brain in younger and older asymptomatic HIV-1 men: Frontal volume changes in the absence of other cortical or diffusion tensor abnormalities. Cortex. 2012;48(2):230-41.

31. Larussa D, Lorenzini P, Cingolani A, Bossolasco S, Grisetti $\mathrm{S}$, Bongiovanni $\mathrm{M}$, et al. Highly active antiretroviral therapy reduces the age-associated risk of dementia in a cohort of older HIV-1-infected patients. AIDS Res Hum Retroviruses. 2006;22(5):386-92.

32. Ciccarelli N, Fabbiani M, Baldonero E, Fanti I, Cauda R, Di Giambenedetto $\mathrm{S}$, et al. Effect of aging and human immunodeficiency virus infection on cognitive abilities. J Am Geriatr Soc. 2012;60(11):2048-55.

33. Ding Y, Lin H, Shen W, Wu Q, Gao M, He N. Interaction effects between HIV and aging on selective neurocognitive impairment. J Neuroimmune Pharmacol. 2017;12(4):661-9.

34. Xiao X, Zeng H, Feng C, Tan H, Wu L, Zhang H, et al. Cognitive impairment among aging people living with HIV on antiretroviral therapy: a cross-sectional study in Hunan. China. J Assoc Nurses 
AIDS Care. 2019. https://doi.org/10.1097/JNC.000000000000012 2.

35. Gawron N, Choinski M, Szymanska-Kotwica B, Pluta A, Sobanska M, Egbert AR, et al. Effects of age, HIV, and HIV-associated clinical factors on neuropsychological functioning and brain regional volume in HIV+ patients on effective treatment. J Neurovirol. 2018. https://doi.org/10.1007/s13365-018-0679-4.

36. Pluta A, Wolak T, Sobanska M, Gawron N, Egbert AR, Szymanska B, et al. HIV and age underlie specific patterns of brain abnormalities and cognitive changes in high functioning patients. Neuropsychology. 2019. https://doi.org/10.1037/neu0000504.

37. Kinai E, Komatsu K, Sakamoto M, Taniguchi T, Nakao A, Igari $\mathrm{H}$, et al. Association of age and time of disease with HIV-associated neurocognitive disorders: a Japanese nationwide multicenter study. J Neurovirol. 2017;23(6):864-74.

38. Pinheiro CA, Mattos Souza LD, Motta JV, Kelbert EF, Martins CS, Souza MS, et al. Aging, neurocognitive impairment and adherence to antiretroviral therapy in human immunodeficiency virus-infected individuals. Braz J Infect Dis. 2016;20(6):599-604.

39. Cysique LA, Maruff P, Bain MP, Wright E, Brew BJ. HIV and age do not substantially interact in HIV-associated neurocognitive impairment. J Neuropsychiatry Clin Neurosci. 2011;23(1):83-9.

40. Kissel EC, Pukay-Martin ND, Bornstein RA. The relationship between age and cognitive function in HIV-infected men. J Neuropsychiatry Clin Neurosci. 2005;17(2):180-4.

41. Wilkie FL, Goodkin K, Khamis I, Van Zuilen MH, Lee D, Lecusay R, et al. Cognitive functioning in younger and older HIV1-infected adults. J Acquir Immune Defic Syndr. 2003;33(SUPPL. 2):S93-S105.

42. Sheppard DP, Woods SP, Bondi MW, Gilbert PE, Massman PJ, Doyle KL. Does older age confer an increased risk of incident neurocognitive disorders among persons living with HIV disease? Clin Neuropsychol. 2015;29(5):656-77.

43. Goodkin K, Miller EN, Cox C, Reynolds S, Becker JT, Martin $\mathrm{E}$, et al. Effect of ageing on neurocognitive function by stage of HIV infection: evidence from the Multicenter AIDS Cohort Study. Lancet HIV. 2017;4(9):e411-e422422.

44. Sacktor N, Skolasky RL, Cox C, Selnes O, Becker JT, Cohen $\mathrm{B}$, et al. Longitudinal psychomotor speed performance in human immunodeficiency virus-seropositive individuals: impact of age and serostatus. J Neurovirol. 2010;16(5):335-41.

45. Seider TR, Luo X, Gongvatana A, Devlin KN, de la Monte SM, Chasman JD, et al. Verbal memory declines more rapidly with age in HIV infected versus uninfected adults. J Clin Exp Neuropsychol. 2014;36(4):356-67.

46. Kupprat SA, Halkitis PN, Perez-Figueroa R, Solomon TM, Ashman T, Kingdon MJ, et al. Age- and education-matched comparison of aging HIV+ men who have sex with men to general population on common neuropsychological assessments. J Health Psychol. 2015;20(9):1175-85.

47. Cohen RA, Gullett JM, Porges EC, Woods AJ, Lamb DG, Bryant VE, et al. Heavy Alcohol Use and Age Effects on HIVAssociated Neurocognitive Function. Alcohol Clin Exp Res. 2019;43(1):147-57.

48. Sandkovsky U, Robertson K, Meza J, High R, Bonasera S, Fisher $\mathrm{C}$, et al. Pilot study of younger and older HIV-infected adults using traditional and novel functional assessments. HIV Clin Trials. 2013;14(4):165-74.

49. Coban H, Robertson K, Smurzynski M, Krishnan S, Kunling W, Boscha RJ. Impact of aging on neurocognitive performance in previously antiretroviral-naive HIV-infected individuals on their first suppressive regimen. AIDS (02699370). 2017;3(11):1565-71.

50. Kim S-H, Miles T, Huber R, Feit MD. Executive cognitive function of older people with HIV/AIDS. J Hum Behav Soc Environ. 2008;18(1):48-63.
51. Vance DE, Wadley VG, Crowe MG, Raper JL, Ball KK. Cognitive and everyday functioning in older and younger adults with and without HIV. Clin Gerontol. 2011;34(5):413-26.

52. Avci G, Loft S, Sheppard DP, Woods SP. The effects of HIV disease and older age on laboratory-based, naturalistic, and selfperceived symptoms of prospective memory: does retrieval cue type and delay interval matter? Neuropsychol Dev Cogn B Aging Neuropsychol Cogn. 2016;23(6):716-43.

53. Scott JC, Woods SP, Carey CL, Weber E, Bondi MW, Grant I. Neurocognitive consequences of HIV infection in older adults: an evaluation of the "cortical" hypothesis. AIDS Behav. 2011;15(6):1187-96.

54. Iudicello JE, Woods SP, Deutsch R, Grant I. Combined effects of aging and HIV infection on semantic verbal fluency: a view of the cortical hypothesis through the lens of clustering and switching. J Clin Exp Neuropsychol. 2012;34(5):476-88.

55. Valcour V, Paul R, Neuhaus J, Shikuma C. The effects of age and HIV on neuropsychological performance. J Int Neuropsychol Soc. 2011;17(1):190-5.

56. Cherner M, Ellis RJ, Lazzaretto D, Young C, Rivera Mindt $\mathrm{M}$, Atkinson JH, et al. Effects of HIV-1 infection and aging on neurobehavioral functioning: preliminary findings. AIDS. 2004;18(SUPPL. 1):S27-S34.

57. Sheppard DP, Iudicello JE, Morgan EE, Kamat R, Clark LR, Avci $\mathrm{G}$, et al. Accelerated and accentuated neurocognitive aging in HIV infection. J Neurovirol. 2017;23(3):492-500.

58. Panos SE, Hinkin CH, Singer EJ, Thames AD, Patel SM, Sinsheimer JS, et al. Apolipoprotein-E genotype and human immunodeficiency virus-associated neurocognitive disorder: the modulating effects of older age and disease severity. Neurobehav HIV Med. 2013;5:11-22.

59. Morgan EE, Woods SP, Delano-Wood L, Bondi MW, Grant I. Intraindividual variability in HIV infection: evidence for greater neurocognitive dispersion in older HIV seropositive adults. Neuropsychology. 2011;25(5):645-54.

60. Foley J, Ettenhofer M, Wright MJ, Siddiqi I, Choi M, Thames $\mathrm{AD}$, et al. Neurocognitive functioning in HIV-1 infection: effects of cerebrovascular risk factors and age. Clin Neuropsychol. 2010;24(2):265-85.

61. Cohen RA, Gullett JM, Porges EC, Woods AJ, Lamb DG, Bryant VE, et al. Heavy alcohol use and age effects on HIV-associated neurocognitive function. Alcoholism. 2019;43(1):147-57.

62. Valcour V, Shikuma C, Shiramizu B, Watters M, Poff P, Selnes O, et al. Higher frequency of dementia in older HIV-1 individuals: the Hawaii aging with HIV-1 Cohort. Neurology. 2004;63(5):822-7.

63. Sacktor N, Skolasky R, Selnes OA, Watters M, Poff P, Shiramizu B, et al. Neuropsychological test profile differences between young and old human immunodeficiency virus-positive individuals. J Neurovirol. 2007;13(3):203-9.

64. Tan IL, Smith BR, Hammond E, Vornbrock-Roosa H, Creighton J, Selnes O, et al. Older individuals with HIV infection have greater memory deficits than younger individuals. J Neurovirol. 2013;19(6):531-6.

65. Van Dyk K, Golub SA, Porter JB, Robin KJ, Kowalczyk WJ, Tomassilli JC, et al. The effects of age and physical health on processing speed in HIV. AIDS Care. 2015;27(10):1326-31.

66. Vance DE, Fazeli PL, Gakumo CA. The impact of neuropsychological performance on everyday functioning between older and younger adults with and without HIV. J Assoc Nurses AIDS Care. 2013;24(2):112-25.

67. Buchner A, Erdfelder E, Faul F, Lang A. G* Power 3.1 manual. Düsseldorf, Germany: Heinrich-Heine-Universitat Dusseldorf. 2017.

68. Janssen RS, Cornblath DR, Epstein LG, Foa RP, McArthur JC, Price RW, et al. Nomenclature and research case definitions for neurologic manifestations of human immunodeficiency virus-type 
1 (HIV-1) infection. Report of a Working Group of the American Academy of Neurology AIDS Task Force. Neurology. 1991;41(6):778-85.

69. Cohen RA, Seider TR, Navia B. HIV effects on age-associated neurocognitive dysfunction: premature cognitive aging or neurodegenerative disease? Alzheimers Res Ther. 2015;7(1):37.

70. Obermeyer Z, Emanuel EJ. Predicting the future-big data, machine learning, and clinical medicine. $\mathrm{N}$ Engl $\mathrm{J}$ Med. 2016;375(13):1216.

71. Brodaty H, Woolf C, Andersen S, Barzilai N, Brayne C, Cheung KS-L, et al. ICC-dementia (International Centenarian Consortium-dementia): an international consortium to determine the prevalence and incidence of dementia in centenarians across diverse ethnoracial and sociocultural groups. BMC Neurol. 2016;16(1):52.

72. Jack CR Jr, Bernstein MA, Fox NC, Thompson P, Alexander G, Harvey D, et al. The Alzheimer's disease neuroimaging initiative (ADNI): MRI methods. J Magn Resonance Imaging. 2008;27(4):685-91.

73. Paul R. Neurocognitive phenotyping of HIV in the era of antiretroviral therapy. Curr HIV/AIDS Rep. 2019;16(3):230-5.

74. Patel SM, Thames AD, Arbid N, Panos SE, Castellon S, Hinkin $\mathrm{CH}$. The aggregate effects of multiple comorbid risk factors on cognition among HIV-infected individuals. J Clin Exp Neuropsychol. 2013;35(4):421-34.

75. Yamada M, Kasagi F, Sasaki H, Masunari N, Mimori Y, Suzuki G. Association between dementia and midlife risk factors: the radiation effects research foundation adult health study. J Am Geriatr Soc. 2003;51(3):410-4.

76. Tedaldi EM, Minniti NL, Fischer T. HIV-associated neurocognitive disorders: the relationship of HIV infection with physical and social comorbidities. BioMed Res Int. 2015;2015:1-13.

77. Egger M, Ekouevi DK, Williams C, Lyamuya RE, Mukumbi H, Braitstein P, et al. Cohort Profile: the international epidemiological databases to evaluate AIDS (IeDEA) in sub-Saharan Africa. Int J Epidemiol. 2012;41(5):1256-64.

78. Hontelez JA, de Vlas SJ, Baltussen R, Newell M-L, Bakker R, Tanser F, et al. The impact of antiretroviral treatment on the age composition of the HIV epidemic in sub-Saharan Africa. AIDS (London England). 2012;26:S19-S30.
79. Joska JA, Dreyer AJ, Nightingale S, Combrinck MI, De Jager CA. Prevalence of HIV-1 Infection in an elderly rural population and associations with neurocognitive impairment. AIDS. 2019;33(11):1765-71.

80. UNAIDS. Global report: UNAIDS report on the global AIDS epidemic 2013. Geneva: Joint United Nations Programme on HIV. AIDS. 2013.

81. Vollmer S, Harttgen K, Alfven T, Padayachy J, Ghys P, Bärnighausen T. The HIV epidemic in sub-Saharan Africa is aging: evidence from the demographic and health surveys in sub-Saharan Africa. AIDS Behav. 2017;21(1):101-13.

82. Eduardo E, Lamb MR, Kandula S, Howard A, Mugisha V, Kimanga D, et al. Characteristics and outcomes among older HIV-positive adults enrolled in HIV programs in four sub-Saharan African countries. PLoS ONE. 2014;9(7):e103864.

83. Habib AG, Yakasai AM, Owolabi LF, Ibrahim A, Habib ZG, Gudaji M, et al. Neurocognitive impairment in HIV-1-infected adults in Sub-Saharan Africa: a systematic review and meta-analysis. Int J Infect Dis. 2013;17(10):e820-e831831.

84. Hendrie HC. Epidemiology of dementia and Alzheimer's disease. Am J Geriatric Psychiatry. 1998;6(2):S3-S18.

85. Brew BJ, Crowe SM, Landay A, Cysique LA, Guillemin G. Neurodegeneration and ageing in the HAART era. J Neuroimmune Pharmacol. 2009;4(2):163-74.

86. Egger K, Schocke M, Weiss E, Auffinger S, Esterhammer R, Goebel G, et al. Pattern of brain atrophy in elderly patients with depression revealed by voxel-based morphometry. Psychiatry Res. 2008;164(3):237-44.

87. Byers AL, Yaffe K. Depression and risk of developing dementia. Nat Rev Neurol. 2011;7(6):323-31.

88. Nightingale S, Winston A, Letendre S, Michael BD, McArthur JC, Khoo S, et al. Controversies in HIV-associated neurocognitive disorders. Lancet Neurol. 2014;13(11):1139-51.

89. Salthouse TA. Methodological assumptions in cognitive aging research. Handb Aging Cogn. 2000;2:467-98.

Publisher's Note Springer Nature remains neutral with regard to jurisdictional claims in published maps and institutional affiliations.

\section{Affiliations}

\section{Htein Linn Aung ${ }^{1,2,3}$ (D) Maral Aghvinian ${ }^{4} \cdot$ Hetta Gouse $^{5} \cdot$ Reuben N. Robbins $^{6} \cdot$ Bruce J. Brew $^{1,3,7} \cdot$ Limin Mao $^{8}$. Lucette A. Cysique ${ }^{1,2,3}$}

Htein Linn Aung

h.aung@amr.org.au; htein_linn.aung@unsw.edu.au

1 Departments of Neurology and HIV Medicine, St Vincent's Hospital and Peter Duncan Neurosciences Unit, St Vincent's Centre for Applied Medical Research (AMR), Level 8, Lowy Packer Building, 405 Liverpool St, Darlinghurst, Sydney, NSW 2010, Australia

2 Neuroscience Research Australia, Sydney, Australia

3 Faculty of Medicine, UNSW, Sydney, Australia

4 Department of Psychology, Fordham University, New York, USA
5 Department of Psychiatry and Mental Health, University of Cape Town, Cape Town, South Africa

6 HIV Center for Clinical and Behavioral Studies, New York State Psychiatric Institute, Department of Psychiatry, Columbia University Vagelos College of Physicians and Surgeons, New York, USA

7 Faculty of Medicine, University of Notre Dame, Sydney, Australia

8 Centre for Social Research in Health, UNSW, Sydney, Australia 